\title{
A comparison of the efficacy of antiangiogenic agents combined with chemotherapy for the treatment of non-small cell lung cancer: a network meta-analysis
}

\author{
Yimin $\mathrm{Li}^{\dagger}$, Yonglin $\mathrm{Yi}^{\dagger}$, Anqi Lin ${ }^{\dagger}$, Peng Luo ${ }^{*}$ (D) and Jian Zhang ${ }^{*}$
}

\begin{abstract}
Objection: To explore the effects of combinations of antiangiogenic agents and chemotherapy agents on non-small cell lung cancer (NSCLC) patients and indirectly compare the therapeutic effect of Endostar combined with chemotherapy and bevacizumab combined with chemotherapy on NSCLC.

Methods: We searched 3 electronic databases: PubMed, Web of Science and the Cochrane Library. The ORRs, HRs and $95 \%$ confidence intervals of OS and PFS were used to compare the efficacy of Endostar combined with chemotherapy and bevacizumab combined with chemotherapy. We use the Bayesian network meta-analysis method to make indirect comparisons and obtain rank probabilities; in addition, we used single-arm meta-analysis to synthesize the existing data.

Results: A total of 29 studies were included in the analysis. Among them, we included a total of 14 interventions. A total of 12,862 patients participated in this analysis. The single-arm meta-analysis showed that the pooled ORR and $95 \% \mathrm{Cl}$ were $0.35(0.31,0.39)$, the pooled HR of OS and $95 \% \mathrm{Cl}$ were $0.89(0.81,0.98)$, and the pooled HR of PFS and $95 \% \mathrm{Cl}$ were $0.67(0.56,0.81)$. According to the results of network meta-analysis, there were no significant differences between the 5 kinds of bevacizumab combined with chemotherapy regimens and the 4 kinds of Endostar combined with chemotherapy regimens for improving ORR and prolonging OS and PFS. The rank probabilities suggested that in terms of ORR, Pla + Pem + Bev was the first-ranked intervention (0.288). Pla + Pem + Endo was the first-ranked intervention for prolonging OS (0.423) and Pla + Gem + Endo was the first-ranked intervention for prolonging PFS (0.302).

Conclusion: Antiangiogenic agents combined with platinum-containing dual drugs can provide benefits to NSCLC patients. In addition, bevacizumab combined with chemotherapy regimens has better theraputic effect on ORR while Endostar combined with chemotherapy may have better effects on OS and PFS for the treatment of NSCLC patients.
\end{abstract}

Keywords: Antiangiogenic agents, Bevacizumab, Endostar, Non-small cell lung cancer, Network meta-analysis

*Correspondence: luopeng@smu.edu.cn; blacktiger@139.com

†Yimin Li, Yonglin Yi and Anqi Lin contributed equally to this study Department of Oncology, Zhujiang Hospital, Southern Medical University,

253 Industrial Avenue, Guangzhou 510282, Guangdong, China

\section{Background}

Lung cancer has the highest incidence rate of cancer in developed countries and is the leading cause of cancerrelated death in the United States [1]. Non-small cell lung cancer (NSCLC) is the main pathological type of lung cancer, accounting for $85 \%$ of all types, and includes

(c) The Author(s) 2020. This article is licensed under a Creative Commons Attribution 4.0 International License, which permits use, sharing, adaptation, distribution and reproduction in any medium or format, as long as you give appropriate credit to the original author(s) and the source, provide a link to the Creative Commons licence, and indicate if changes were made. The images or other third party material in this article are included in the article's Creative Commons licence, unless indicated otherwise in a credit line to the material. If material is not included in the article's Creative Commons licence and your intended use is not permitted by statutory regulation or exceeds the permitted use, you will need to obtain permission directly from the copyright holder. To view a copy of this licence, visit http://creativeco mmons.org/licenses/by/4.0/. The Creative Commons Public Domain Dedication waiver (http://creativecommons.org/publicdomain/ zero/1.0/) applies to the data made available in this article, unless otherwise stated in a credit line to the data. 
squamous cell carcinoma, adenocarcinoma, large cell carcinoma and adenosquamous carcinoma. Angiogenesis, which was proposed by Folkman in 1971 [2] and listed as one of the hallmarks of tumors by Hanahan [3], plays an important role in the occurrence and development of tumors. Angiogenesis is related to the proliferation, invasion and metastasis of tumors [4]. Many molecules, such as vascular endothelial growth factor (VEGF), platelet-derived growth factor (PDGF), transforming growth factor- $\beta$ (TGF- $\beta$ ), fibroblast growth factor (FGF) and other important molecules, participate in the process of angiogenesis [5]. Currently, with various antiangiogenic agents being approved for cancer treatment, antiangiogenic therapy for NSCLC has attracted increasing attention.

Bevacizumab is a humanized monoclonal antibody with high affinity to VEGF [6]. The VEGF family includes VEGF-A, VEGF-B, VEGF-C, VEGF-D and placental growth factor, which have been suggested to be overexpressed in NSCLC, providing necessary conditions for angiogenesis [7]. Bevacizumab has a high affinity and specificity for VEGF, and therefore, it can inhibit the growth of NSCLC [6], which provides a premise for it to become an agent for the treatment of NSCLC. The results of E4599, which is a randomized, controlled, multicenter clinical trial, show that bevacizumab combined with carboplatin and paclitaxel can significantly improve the response rate and prolong progression-free survival (PFS) and overall survival (OS) compared with carboplatin and paclitaxel alone for patients with unresectable and advanced non-squamous cell carcinoma who were chemotherapy naive [8]. Based on this finding, in 2005, the Food and Drug Administration approved bevacizumab combined with carboplatin and paclitaxel for the first-line treatment of advanced non-squamous cell carcinoma [9]. However, at present, only bevacizumab combined with cisplatin and paclitaxel has been approved for NSCLC treatment, and the differences in outcomes of bevacizumab combined with different chemotherapies have not been clearly determined.

Endostatin is a kind of natural protein that was first isolated and extracted by Judah Folkman in mouse tumor strains and showed the strong antiangiogenic and tumor growth inhibition effects [10]. Endostatin has a wide antitumor spectrum [11]. The specific antiangiogenic mechanism has not been fully elucidated at present. The main mechanism of the antitumor effect is that endostatin acts on the VEGF receptor KDR/Flk-1 and inhibits the signal transduction of VEGF, which therefore inhibits angiogenesis [12]. Endostar is a recombinant human endostatin with 9 added amino acids (MGGSHHHHH) [13] to maintain stability and a long half-life. A randomized, double-blind, controlled, multicenter phase III clinical trial from China showed that the combination of Endostar with vinorelbine and cisplatin can significantly improve the response rate, median time to progression (TTP), and quality of life of patients with advanced NSCLC compared with vinorelbine and cisplatin alone. There were no significant differences in adverse events, but its cardiotoxicity needs further attention [14]. Endostar was approved by the China Food and Drug Administration in 2005 for the treatment of NSCLC [15]. However, at present, only one phase 3 clinical trial has shown that the efficacy of Endostar combined chemotherapy, and the advantages of Endostar combined with other chemotherapeutics need more rigorous clinical trials for confirmation.

Currently, it is still not clear that antiangiogenic drugs combined with which chemotherapy regime can provide the most benefit to NSCLC patients. Besides, although the results from phase 3 clinical trials showed that NSCLC patients can benefit from bevacizumab combined with chemotherapy and Endostar combined with chemotherapy for first-line treatment, there is a lack of head-to-head clinical trials of the two schemes. Therefore, we used the Bayesian network meta-analysis method to indirectly compare the efficacy of bevacizumab combined with chemotherapy and Endostar combined with chemotherapy in the treatment of NSCLC. In addition, we used single-arm meta-analysis to synthesize published clinical trial results to comprehensively evaluate the therapeutic effect of bevacizumab combined with chemotherapy and Endostar combined with chemotherapy on NSCLC patients.

\section{Materials and methods}

\section{Search strategy and eligibility criteria}

On May 1, 2020, we used the following key words to retrieve literature from the PubMed, Web of Science and the Cochrane Library databases: "Anti-VEGF Humanized Monoclonal Antibody", "anti VEGF monoclonal antibody", "rhuMAb-VEGF"; "Endostar", "recombinant human endostatin", "Rh endostatin", "YH-16"; "Avastin", "bevacizumab"; "Cyramza", "Ramucirumab", "Brigatinib", "Alunbrig", "Cabozantinib", "Cabometyx", "Cometriq"; "non small cell lung cancer", "Lung cancer" and "NSCLC". All MeSH terms and entry terms were used to achieve a comprehensive search. In this search, there were no restrictions on the language or publication date.

Inclusion and exclusion criteria were established for the purpose of our analysis. The inclusion criteria were as follows: the study type was a clinical trial or prospective study; the study population was NSCLC patients who had not received chemotherapy or NSCLC patients who had completed previous chemotherapy for more than or equal to 4 weeks; the interventions were bevacizumab 
combined with chemotherapy, Endostar combined with chemotherapy or chemotherapy alone; the study design involved studies with at least two arms and included at least the above two interventions or included the same intervention in the same study but used different chemotherapy agents; and studies that provided at least one of the following outcome measures: objective response rate (ORR), overall survival (OS), and progression free survival (PFS), studies that provided the event rate of adverse events after treatment were also included. The exclusion criteria included articles that could not be provided in full-text and non-English articles; and the following article types were also excluded: review articles, case reports, meeting abstracts, meta-analyses, cell animal experiments, etc. Three reviewers (YL, YY and AL) independently performed preliminary screening according to the titles and abstracts and then confirmed whether to include the studies by reading the full texts according to the inclusion and exclusion criteria. Any disputes regarding the included studies were resolved through discussion with the fourth reviewer (PL).

\section{Data extraction}

Three reviewers (YL, YY and AL) used a previously designed data extraction table to extract data independently. The extracted data included the general data of the study (such as the first author, study year, proportion of males, treatment plan, performance status, proportion of squamous cell carcinoma), the event rate of adverse events, the ORRs, hazard ratios (HRs) and 95\% confidence intervals (CIs) of OS and PFS. If the HR and 95\% CI were not provided in the study, we used Tierney's method to estimate them [16]. Any disputes or inconsistencies were discussed with the fourth reviewer (PL).

\section{Quality assessment}

Three reviewers (YL, YY and AL) independently evaluated the quality of the research methods of the included studies. The Cochrane Collaboration's risk of bias (ROB) tool was used to evaluate the quality of randomized controlled trials [17], and the Newcastle Ottawa Scale (NOS) was used to evaluate the quality of nonrandomized controlled trials and prospective studies [18]. For randomized controlled trials, if there were more than four "low risk" domains based on the ROB tool, the study was considered to have "high quality"; if there were two or more "low risk" domains, the study was considered to have "moderate quality"; and finally, if there were less than two "low risk" domains or more than one "high risk" domain, the study was considered to have "low quality". For nonrandomized controlled trials, if the total NOS score was 7-9, the study was considered to have "high quality"; if the total NOS score was less than 4, the study was considered to have "low quality"; and if the total NOS score was $4-6$, the study was considered to have "moderate quality". Similarly, any disputes and inconsistencies were discussed with the fourth reviewer (PL).

\section{Statistical analysis}

R 3.5.1 software (The R Foundation, Vienna, Austria) was used for Bayesian meta-analysis. For the binary variable (ORR) and time-to-event data (HRs of OS and PFS), we used the "gemtc" package and "JAGS" package of $\mathrm{R}$ software, using the Markov chain Monte Carlo method [19] to simulate four different chains, with 40,000 iterations, 160,000 burn-ins and a thinning interval of 10 . The Bayesian network meta-analysis was carried out so that each intervention could be compared indirectly, and the rank probabilities of various interventions can be obtained. For the comparison of ORR, the results are shown as odds ratios (ORs) and 95\% credible intervals (CrIs); for the comparison of OS and PFS, the results are shown as HRs and 95\% CrIs, and the results of all CrIs were bilateral. In addition, to ensure the credibility of the results, we used the "gemtc" package and "JAGS" package in R 3.5.1 software to analyze the ORR and HR of OS and PFS by nodesplit analysis to explore the consistency between direct comparisons and indirect comparisons. $\mathrm{P}>0.05$ indicates that the difference between the direct comparison and the indirect comparison was not statistically significant. $\mathrm{P}<0.05$ indicates that the difference between the direct comparison and the indirect comparison was statistically significant. Heterogeneity analysis was used to compare the degree of heterogeneity in the research results. When the $\mathrm{P}$ value was more than 0.1 and $\mathrm{I}^{2}$ was less than $50 \%$, the heterogeneity was not statistically significant. When the P value was less than 0.1 and $\mathrm{I}^{2}$ was more than $50 \%$, the heterogeneity was statistically significant.

Review Manager 5.3.4 software (Cochrane Library, Oxford, UK) and STATA 14.0 software (Stata Corp., College Station, TX) were used for single-arm meta-analysis. The ORR, OS, PFS, HRs of OS and PFS and the event rate of adverse events were used to obtain pooled results. The statistical model was selected according to the degree of heterogeneity. When the $\mathrm{P}$ value was more than 0.1 and $\mathrm{I}^{2}$ was less than $50 \%$, no statistically significant heterogeneity was indicated, so a fixed effects model was used. When the $\mathrm{P}$ value was less than 0.1 and $\mathrm{I}^{2}$ was more than $50 \%$, statistically significant heterogeneity was indicated, so a random effect models was used. At the same time, we used RevMan to evaluate publication bias intuitively through funnel plots and STATA to evaluate publication bias quantitatively through Begg's and Egger's tests. In addition, STATA was used to generate a network plot 
to describe the number of patients included in the study and the number of head-to-head comparisons.

\section{Results}

\section{Characteristics of the included studies}

According to the search strategy, we retrieved 10,650 articles in PubMed, Web of Science and the Cochrane Library. Sun Yan et al. provided inadequate information [47] for our network meta-analysis, and therefore, we e-mailed the corresponding authors for further information. Finally, the author provided us with more detailed results of a phase 4 clinical trial of Endostar as additional sources for retrieval. Finally, a total of 29 articles were included in our study, including 4 nonrandomized controlled trials and 25 randomized controlled trials. The screening process of the articles is shown as a flow diagram in Fig. 1. The general characteristics of the patients in each study are shown in Table 1 . The details of quality assessment are shown in Additional file 1: Figure S1.

\section{Network meta-analysis of the outcome measures}

To facilitate the study, we summarized the interventions involved in the articles as follows: platinum + gemcitabine + Endostar $\quad(\mathrm{Pla}+\mathrm{Gem}+$ Endo $)$, platinum + gemcitabine + bevaczumab $(\mathrm{Pla}+\mathrm{Gem}+\mathrm{Bev})$, platinum + gemcitabine $(\mathrm{Pla}+\mathrm{Gem})$, platinum + paclitaxel + Endostar $\quad(\mathrm{Pla}+\mathrm{Pac}+$ Endo $)$, platinum + paclitaxel + bevacizumab $\quad(\mathrm{Pla}+\mathrm{Pac}+\mathrm{Bev})$, platinum + paclitaxel (Pla + Pac $), \quad$ platinum + pemetrexed + bevacizumab $\quad(\mathrm{Pla}+\mathrm{Pem}+\mathrm{Bev})$, platinum + pemetrexed $(\mathrm{Pla}+\mathrm{Pem})$, pemetrexed + bevacizumab $($ Pem + Bev), vinorelbine + platinum $($ Vin + Pla $)$, vinorelbine + platinum + Endostar $\quad($ Vin + Pla + Endo $)$, gemcitabine + bevacizumab (Gem $+\mathrm{Bev})$ and platinum + pemetrexed + Endostar $\quad(\mathrm{Pla}+\mathrm{Pem}+$ Endo $) . \quad \mathrm{A}$ total of 12,862 patients were treated with these interventions. The sample size of patients included in each intervention and the number of head-to-head comparisons were roughly described as a network plot, as shown in Fig. 2. The results of the Bayesian meta-analysis of ORR, OS and PFS are summarized in Fig. 3. The analyses of OS and PFS were not fully included for all interventions because some studies were unable to provide data related to ORR, OS or PFS.

For ORR, there were no significant differences (the CrI of the OR value included 1 ) in the improvement of the ORR of NSCLC patients between 4 different Endostar combined with chemotherapy regimens $(\mathrm{Pla}+\mathrm{Gem}+$ Endo, $\mathrm{Pla}+\mathrm{Pac}+$ Endo, and $\mathrm{Vin}+\mathrm{Pla}+$ Endo, Pla + Pem + Endo) and 5 bevacizumab combined with chemotherapy regimens $(\mathrm{Pla}+\mathrm{Gem}+\mathrm{Bev}, \quad \mathrm{Pla}+\mathrm{Pac}+\mathrm{Bev}, \quad \mathrm{Pla}+\mathrm{Pem}+\mathrm{Bev}$, $\mathrm{Pem}+\mathrm{Bev}$ and $\mathrm{Gem}+\mathrm{Bev})$. There were no significant differences among the 4 Endostar combined with chemotherapy regimens. However, some Endostar combined with chemotherapy regimens had significant advantages over some chemotherapy regimens based on platinum. Pla $+\mathrm{Gem}+$ Endo was superior to $\mathrm{Pla}+\mathrm{Gem}$ [OR: $0.47(0.25,0.90)], \mathrm{Pla}+\mathrm{Pac}$ [OR: $0.41(0.21,0.78)$, and Vin +Pla [OR: $0.42(0.19,0.94)]$, and there were no significant differences between $\mathrm{Pla}+\mathrm{Pac}+$ Endo, Vin + Pla + Endo and platinum-based chemotherapy, except Pla + Pac vs Vin + Pla + Endo [OR: $2.3(1.1,4.6)]$. For the comparison among bevacizumab combined with chemotherapy regimens, only $\mathrm{Pla}+\mathrm{Pem}+\mathrm{Bev}$ was superior to Pem + Bev [OR: $0.57(0.34,0.96)]$. Except for $\mathrm{Pem}+\mathrm{Bev}$ and Gem + Bev, bevacizumab combined with chemotherapy was superior to some chemotherapy regimens: $\mathrm{Pla}+\mathrm{Gem}+\mathrm{Bev}$ was superior to $\mathrm{Pla}+\mathrm{Gem}[\mathrm{OR}$ : 0.57 (0.34, 0.98)] and Pla + Pac [OR: $0.50(0.27,0.88)$ ]; $\mathrm{Pla}+\mathrm{Pac}+\mathrm{Bev}$ was better than Pla + Pac [OR: 0.39 (0.30, $0.51)], \mathrm{Pla}+\mathrm{Pem}$ [OR: $0.56(0.40,0.81)]$, and Vin + Pla [OR: $0.41(0.23,0.72)$ ]; and Pla + Pem + Bev was better than Vin + Pla [OR: $0.39(0.19,0.83)]$. Interestingly, for the improvement of ORR, Endostar combined with chemotherapy regimens $(\mathrm{Pla}+\mathrm{Gem}+$ Endo, $\mathrm{Pla}+\mathrm{Pac}+$ Endo, Vin $+\mathrm{Pla}+$ Endo and Pla + Pem + Endo) and bevacizumab combined with chemotherapy regimens $(\mathrm{Pla}+\mathrm{Gem}+\mathrm{Bev}, \mathrm{Pla}+\mathrm{Pac}+\mathrm{Bev}, \mathrm{Pla}+\mathrm{Pem}+\mathrm{Bev}$ and Pem $+\mathrm{Bev})$, were significantly better than Pem alone, with ORs and 95\% CrIs of $0.17(0.049,0.58), 0.21(0.061$, $0.67), 5.4(1.5,20.0), 5.2(1.3,22.0), 0.16(0.058,0.44)$, $0.23(0.064,0.77)$, and $0.16(0.062,0.38), 3.6(1.6,8.9)$, respectively.

For the time-to-event data of OS, there were no significant differences between the Endostar combined with chemotherapy regimens and the bevacizumab combined with chemotherapy regimens. For the bevacizumab combined with chemotherapy regimens, $\mathrm{Pla}+\mathrm{Pac}+\mathrm{Bev}$ was significantly better at prolonging OS than Pla + Gem [HR: 0.83 (0.71, 0.97)]; similarly, compared with $\mathrm{Pla}+\mathrm{Pac}$, $\mathrm{Pla}+\mathrm{Pac}+\mathrm{Bev}$ can also significantly reduce the HR [HR: $1.2(1.1,1.4)](\mathrm{Pla}+\mathrm{Pac}$ vs $\mathrm{Pla}+\mathrm{Pac}+\mathrm{Bev})$.

The results of rank probabilities for ORR, OS and PFS were shown as heat maps in Fig. 4 (See more detailed data in Additional file 1: Table S1). For the time-to-event data of PFS, there were no significant differences between the Endostar combined with chemotherapy regimens and the bevacizumab combined with chemotherapy regimens, similarly. And for the bevacizumab combined with chemotherapy regimens, $\mathrm{Pla}+\mathrm{Pac}+\mathrm{Bev}$ was superior to $\mathrm{Pla}+$ Gem [HR: $0.57(0.42,0.79)]$ and Pla + Pac [HR: 1.9 $(1.5,2.4)](\mathrm{Pla}+\mathrm{Pac}$ vs $\mathrm{Pla}+\mathrm{Pac}+\mathrm{Bev})$.

According to the results of network meta-analysis for ORR, Pla $+\mathrm{Pem}+\mathrm{Bev}$ was the most likely best intervention (0.288) and $\mathrm{Pla}+\mathrm{Pac}+\mathrm{Bev}(0.255)$ was 


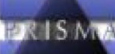

\section{PRISMA 2009 Flow Diagram}
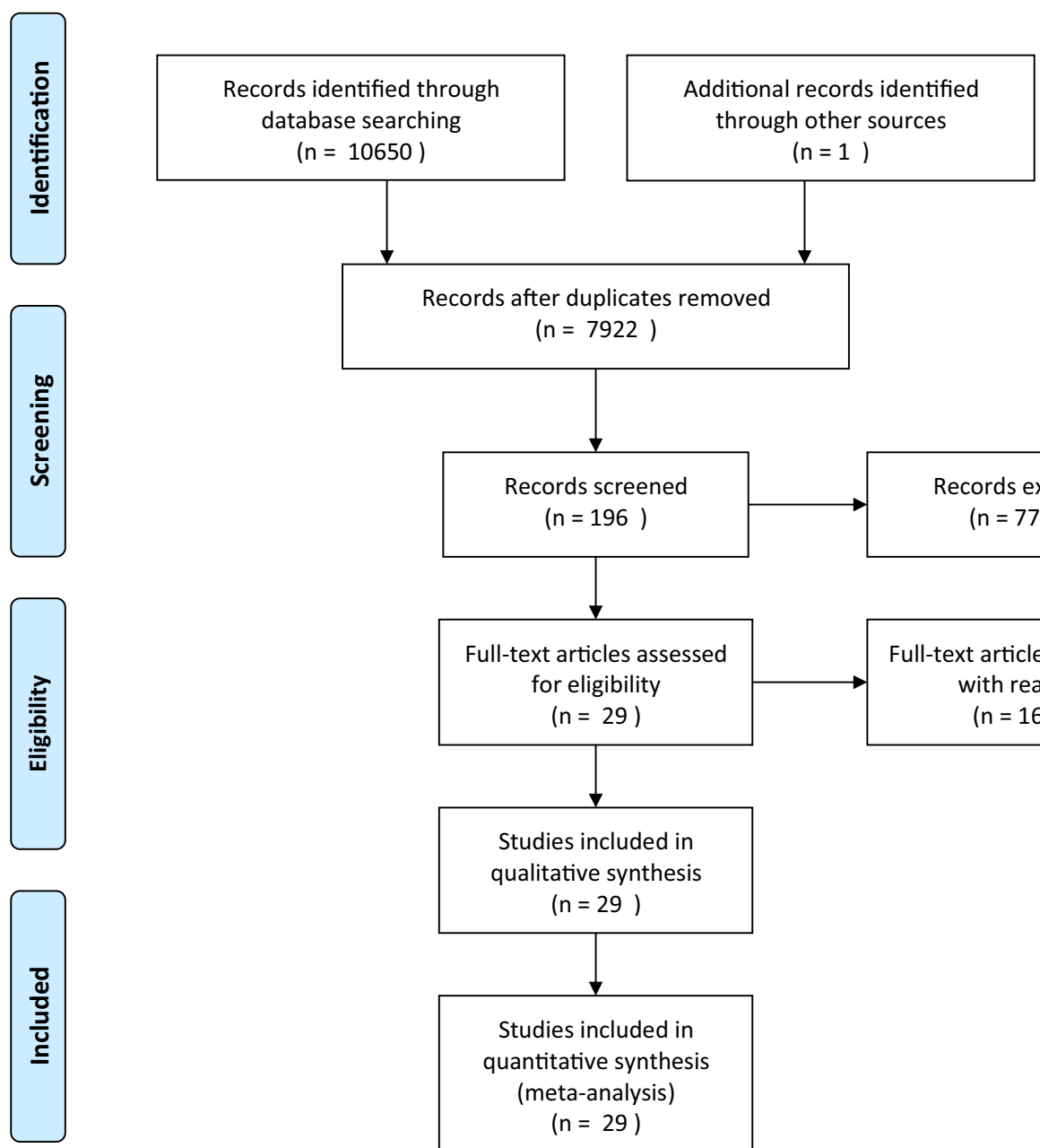

$$
(n=1)
$$

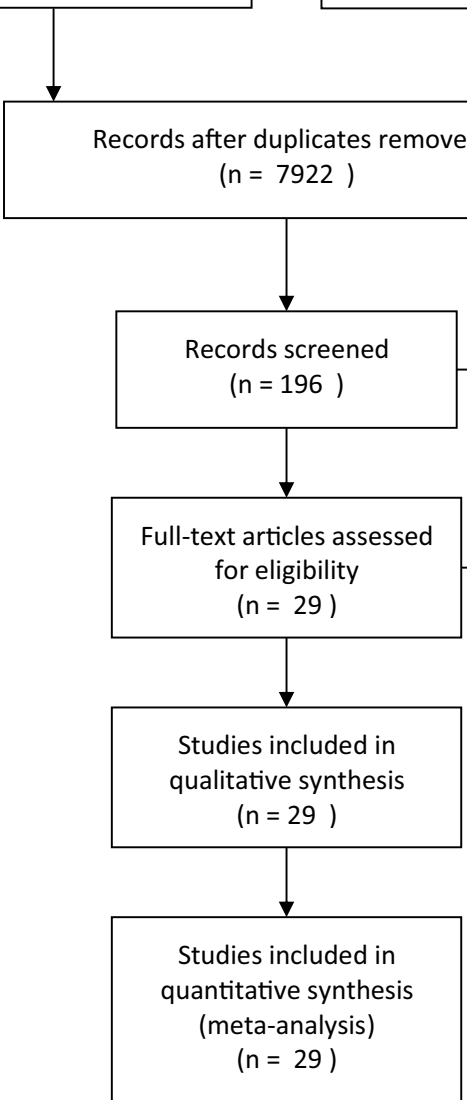

From: Moher D, Liberati A, Tetzlaff J, Altman DG, The PRISMA Group (2009). Preferred Reporting /tems for Systematic Reviews and MetaAnalyses: The PRISMA Statement. PLoS Med 6(7): e1000097. doi:10.1371/journal.pmed1000097

For more information, visit www.prisma-statement.org.

Fig. 1 The flow diagram of the study selection process for the network meta-analysis

the most likely second best intervention. In terms of the time-to-event data of OS, Pla + Pem + Endo was the most likely best intervention (0.423), Pem $+\mathrm{Bev}$ (0.219) was the most likely second best intervention. And for the time-to-event data of PFS, the most likely best intervention was Pla $+\mathrm{Gem}+$ Endo (0.302), and the most likely second best intervention was $\mathrm{Pla}+\mathrm{Pac}+\mathrm{Bev}(0.340)$.

\section{Nodesplit and heterogeneity analyses}

The results of nodesplit analysis and heterogeneity were shown in Fig. 5. To test the consistency and heterogeneity 
Table 1 General characteristics and quality assessment of the included studies

\begin{tabular}{|c|c|c|c|c|c|c|c|c|}
\hline Study ID & Treatment & Sample size & Median age & Male (\%) & Stage IV (\%) & SCC (\%) & $\mathrm{PS}=2$ & Quality assesment \\
\hline \multirow[t]{2}{*}{ Boutsikou [20] } & $\begin{array}{l}\text { Docetaxel }\left(100 \mathrm{mg} / \mathrm{m}^{2}\right)+\text { Carboplatin } \\
\left(5 \mathrm{mg} / \mathrm{mL}^{*} \mathrm{~min}\right)+\text { Bevacizumab } \\
(7.5 \mathrm{mg} / \mathrm{kg})\end{array}$ & 56 & 63 & 80.4 & 73.2 & 0 & 0 & Moderate \\
\hline & $\begin{array}{l}\text { Docetaxel }\left(100 \mathrm{mg} / \mathrm{m}^{2}\right)+\text { Carboplatin } \\
\left(5 \mathrm{mg} / \mathrm{mL}^{*} \mathrm{~min}\right)\end{array}$ & 61 & 65 & 85.2 & 83.6 & 0 & 0 & \\
\hline \multirow[t]{2}{*}{ Fukuda [21] } & $\begin{array}{l}\text { Bevacizumab }(15 \mathrm{mg} / \mathrm{kg})+\text { pem- } \\
\text { etrexed }\left(500 \mathrm{mg} / \mathrm{m}^{2}\right)\end{array}$ & 20 & 78.5 & 55 & 75 & 0 & 0 & Moderate \\
\hline & pemetrexed $\left(500 \mathrm{mg} / \mathrm{m}^{2}\right)$ & 20 & 77.5 & 60 & 75 & 0 & 0 & \\
\hline \multirow[t]{2}{*}{ Galetta [22] } & $\begin{array}{l}\text { Paclitaxel }\left(200 \mathrm{mg} / \mathrm{m}^{2}\right)+\text { Carboplatin } \\
\left(6 \mathrm{mg} / \mathrm{mL} L^{*} \mathrm{~min}\right)+\text { Bevacizumab } \\
(15 \mathrm{mg} / \mathrm{kg})\end{array}$ & 58 & 62 & 77.6 & 93.1 & 0 & 0 & High \\
\hline & $\begin{array}{l}\text { Pemetrexed }\left(500 \mathrm{mg} / \mathrm{m}^{2}\right)+\text { Cisplatin } \\
\left(75 \mathrm{mg} / \mathrm{m}^{2}\right)\end{array}$ & 60 & 60 & 70 & 95 & 0 & 0 & \\
\hline \multirow[t]{2}{*}{ Gronberg [23] } & $\begin{array}{l}\text { Gemcitabine }\left(1,000 \mathrm{mg} / \mathrm{m}^{2}\right)+\text { Carbo- } \\
\text { platin }\left(6 \mathrm{mg} / \mathrm{mL}^{*} \mathrm{~min}\right)\end{array}$ & 217 & 66 & 59.0 & 71.9 & 23.0 & 22.6 & High \\
\hline & $\begin{array}{l}\text { Pemetrexed }\left(500 \mathrm{mg} / \mathrm{m}^{2}\right)+\text { Carbopl- } \\
\text { atin }\left(6 \mathrm{mg} / \mathrm{mL}^{*} \mathrm{~min}\right)\end{array}$ & 219 & 64 & 56.2 & 71.2 & 26.0 & 21.5 & \\
\hline \multirow[t]{2}{*}{ Han [24] } & $\begin{array}{l}\text { Paclitaxel }\left(175 \mathrm{mg} / \mathrm{m}^{2} \text { on day } 1\right)+\text { Car- } \\
\text { boplatin }\left(5 \mathrm{mg} / \mathrm{mL}^{*} \mathrm{~min} \text { on day }\right. \\
\text { 1)+ Endostar }\left(7.5 \mathrm{mg} / \mathrm{m}^{2} / \mathrm{d} \text { on days }\right. \\
8 \text { and } 21)\end{array}$ & 61 & 57 & 80.3 & 70.5 & 37.7 & 6.6 & High \\
\hline & $\begin{array}{l}\text { Paclitaxel }\left(175 \mathrm{mg} / \mathrm{m}^{2} \text { on day } 1\right)+\text { Car- } \\
\text { boplatin }\left(5 \mathrm{mg} / \mathrm{mL}^{*} \mathrm{~min} \text { on day }\right. \\
\text { 1)+ Endostar }\left(7.5 \mathrm{mg} / \mathrm{m}^{2} / \mathrm{d} \text { on days }\right. \\
8 \text { and } 21)\end{array}$ & 61 & 58 & 62.3 & 59 & 23 & 3.3 & \\
\hline \multirow[t]{2}{*}{ Johnson [25] } & $\begin{array}{l}\text { Paclitaxel }\left(200 \mathrm{mg} / \mathrm{m}^{2}\right)+\text { Carboplatin } \\
\left(6 \mathrm{mg} / \mathrm{mL}^{*} \mathrm{~min}\right)+\text { Bevacizumab } \\
(15 \mathrm{mg} / \mathrm{kg})\end{array}$ & 35 & 57 & 45.7 & 80 & 8.6 & 11.4 & Moderate \\
\hline & $\begin{array}{l}\text { Paclitaxel }\left(200 \mathrm{mg} / \mathrm{m}^{2}\right)+\text { Carboplatin } \\
\left(6 \mathrm{mg} / \mathrm{mL}^{*} \mathrm{~min}\right)\end{array}$ & 32 & 58 & 75 & 81.3 & 21.9 & 6.3 & \\
\hline \multirow[t]{2}{*}{ Niho [26] } & $\begin{array}{l}\text { Paclitaxel }\left(200 \mathrm{mg} / \mathrm{m}^{2}\right)+\text { Carboplatin } \\
\left(6 \mathrm{mg} / \mathrm{mL} \mathrm{L}^{*} \mathrm{~min}\right)+\text { Bevacizumab } \\
(15 \mathrm{mg} / \mathrm{kg})\end{array}$ & 121 & 61 & 63.6 & 68.6 & 0 & 0 & High \\
\hline & $\begin{array}{l}\text { Paclitaxel }\left(200 \mathrm{mg} / \mathrm{m}^{2}\right)+\text { Carboplatin } \\
\left(6 \mathrm{mg} / \mathrm{mL}^{*} \mathrm{~min}\right)\end{array}$ & 59 & 60 & 64.4 & 71.2 & 0 & 0 & \\
\hline \multirow[t]{2}{*}{ Patel [27] } & $\begin{array}{l}\text { Pemetrexed }\left(500 \mathrm{mg} / \mathrm{m}^{2}\right)+\text { Carbopl- } \\
\text { atin }\left(6 \mathrm{mg} / \mathrm{mL}^{*} \mathrm{~min}\right)+\text { Bevacizumab } \\
(15 \mathrm{mg} / \mathrm{kg})\end{array}$ & 472 & 65 & 53.2 & 89.8 & 0 & 0 & High \\
\hline & $\begin{array}{l}\text { Paclitaxel }\left(200 \mathrm{mg} / \mathrm{m}^{2}\right)+\text { Carboplatin } \\
\left(6 \mathrm{mg} / \mathrm{mL} \mathrm{L}^{*} \mathrm{~min}\right)+\text { Bevacizumab } \\
(15 \mathrm{mg} / \mathrm{kg})\end{array}$ & 467 & 65 & 53.3 & 89.9 & 0 & 0 & \\
\hline \multirow[t]{2}{*}{ Pereira [28] } & $\begin{array}{l}\text { Pemetrexed }\left(500 \mathrm{mg} / \mathrm{m}^{2}\right)+\text { Carbopl- } \\
\text { atin }\left(5 \mathrm{mg} / \mathrm{mL}^{*} \mathrm{~min}\right)\end{array}$ & 106 & 60 & 60.4 & 84.0 & 0 & 14.2 & Moderate \\
\hline & $\begin{array}{l}\text { Docetaxel }\left(75 \mathrm{mg} / \mathrm{m}^{2}\right)+\text { Carboplatin } \\
\left(5 \mathrm{mg} / \mathrm{mL}^{*} \mathrm{~min}\right)\end{array}$ & 105 & 59 & 47.6 & 78.1 & 0 & 16.2 & \\
\hline \multirow[t]{2}{*}{ Reck [29] } & $\begin{array}{l}\text { Gemcitabine }\left(1,250 \mathrm{mg} / \mathrm{m}^{2}\right)+\text { Cispl- } \\
\text { atin }\left(80 \mathrm{mg} / \mathrm{m}^{2}\right)+\text { Bevacizumab } \\
(15 \mathrm{mg} / \mathrm{kg})\end{array}$ & 351 & 59 & 62.4 & 76.6 & 0 & 0 & High \\
\hline & $\begin{array}{l}\text { Gemcitabine }\left(1,250 \mathrm{mg} / \mathrm{m}^{2}\right)+\text { Cispl- } \\
\text { atin }\left(80 \mathrm{mg} / \mathrm{m}^{2}\right)\end{array}$ & 347 & 59 & 64.3 & 76.7 & 0 & - & \\
\hline \multirow[t]{2}{*}{ Sandler [8] } & $\begin{array}{l}\text { Paclitaxel }\left(200 \mathrm{mg} / \mathrm{m}^{2}\right)+\text { Carboplatin } \\
\left(6 \mathrm{mg} / \mathrm{mL} \mathrm{L}^{*} \mathrm{~min}\right)+\text { Bevacizumab } \\
(15 \mathrm{mg} / \mathrm{kg})\end{array}$ & 417 & 56 & 50.4 & 74.3 & 0 & 0 & High \\
\hline & $\begin{array}{l}\text { Paclitaxel }\left(200 \mathrm{mg} / \mathrm{m}^{2}\right)+\text { Carboplatin } \\
\left(6 \mathrm{mg} / \mathrm{mL}^{*} \mathrm{~min}\right)\end{array}$ & 433 & 58 & 58.4 & 77.8 & 0 & 0 & \\
\hline
\end{tabular}


Table 1 (continued)

\begin{tabular}{|c|c|c|c|c|c|c|c|c|}
\hline Study ID & Treatment & Sample size & Median age & Male (\%) & Stage IV (\%) & SCC (\%) & $\mathrm{PS}=2$ & Quality assesment \\
\hline \multirow[t]{2}{*}{ Scagliotti [30] } & $\begin{array}{l}\text { Pemetrexed }\left(500 \mathrm{mg} / \mathrm{m}^{2}\right)+\text { Cisplatin } \\
\left(75 \mathrm{mg} / \mathrm{m}^{2}\right)\end{array}$ & 862 & 61 & 70.2 & 76.2 & 40.6 & 0 & Moderate \\
\hline & $\begin{array}{l}\text { Gemcitabine }\left(1,000 \mathrm{mg} / \mathrm{m}^{2}\right)+\text { Cispl- } \\
\text { atin }\left(75 \mathrm{mg} / \mathrm{m}^{2}\right)\end{array}$ & 863 & 61 & 75.7 & 75.7 & 43.5 & 0 & \\
\hline \multirow[t]{3}{*}{ Scagliotti [31] } & $\begin{array}{l}\text { Gemcitabine }\left(1,250 \mathrm{mg} / \mathrm{m}^{2}\right)+\text { Cispl- } \\
\text { atin }\left(75 \mathrm{mg} / \mathrm{m}^{2}\right)\end{array}$ & 205 & 63 & 81.5 & 81.5 & 32.7 & 5.4 & Moderate \\
\hline & $\begin{array}{l}\text { Vinorelbine }\left(25 \mathrm{mg} / \mathrm{m}^{2}\right)+\text { Cisplatin } \\
\left(75 \mathrm{mg} / \mathrm{m}^{2}\right)\end{array}$ & 201 & 62 & 78.1 & 81.1 & 27.4 & 8.0 & \\
\hline & $\begin{array}{l}\text { Paclitaxel }\left(225 \mathrm{mg} / \mathrm{m}^{2}\right)+\text { Cisplatin } \\
\left(75 \mathrm{mg} / \mathrm{m}^{2}\right)\end{array}$ & 201 & 62 & 75.6 & 81.6 & 32.3 & 8.5 & \\
\hline \multirow[t]{4}{*}{ Schiller [32] } & $\begin{array}{l}\text { Gemcitabine }\left(1,000 \mathrm{mg} / \mathrm{m}^{2}\right)+\text { Cispl- } \\
\text { atin } \\
\left(100 \mathrm{mg} / \mathrm{m}^{2}\right)\end{array}$ & 288 & 64 & 62.0 & 89.0 & 22.3 & 6.0 & High \\
\hline & $\begin{array}{l}\text { Paclitaxel }\left(135 \mathrm{mg} / \mathrm{m}^{2}\right)+\text { Cisplatin } \\
\left(75 \mathrm{mg} / \mathrm{m}^{2}\right)\end{array}$ & 288 & 62 & 64.0 & 86.0 & 26.8 & 5.0 & \\
\hline & $\begin{array}{l}\text { Docetaxel }\left(75 \mathrm{mg} / \mathrm{m}^{2}\right)+\text { Cisplatin } \\
\left(75 \mathrm{mg} / \mathrm{m}^{2}\right)\end{array}$ & 289 & 63 & 62.0 & 86.0 & 25.0 & 6.0 & \\
\hline & $\begin{array}{l}\text { Paclitaxel }\left(225 \mathrm{mg} / \mathrm{m}^{2}\right)+\text { Carboplatin } \\
\left(6 \mathrm{mg} / \mathrm{mL}^{*} \mathrm{~min}\right)\end{array}$ & 290 & 63 & 63.0 & 86.0 & 25.9 & 5.0 & \\
\hline \multirow[t]{2}{*}{ Smit [33] } & $\begin{array}{l}\text { Gemcitabine }\left(1,250 \mathrm{mg} / \mathrm{m}^{2}\right)+\text { Cispl- } \\
\text { atin }\left(80 \mathrm{mg} / \mathrm{m}^{2}\right)\end{array}$ & 160 & 57 & 70.6 & 78.8 & 25.6 & 11.3 & High \\
\hline & $\begin{array}{l}\text { Paclitaxel }\left(175 \mathrm{mg} / \mathrm{m}^{2}\right)+\text { Cisplatin } \\
\left(80 \mathrm{mg} / \mathrm{m}^{2}\right)\end{array}$ & 159 & 57 & 59.7 & 81.8 & 18.9 & 11.9 & \\
\hline \multirow[t]{2}{*}{ Soria [34] } & $\begin{array}{l}\text { Paclitaxel }\left(200 \mathrm{mg} / \mathrm{m}^{2}\right)+\text { Carboplatin } \\
\left(6 \mathrm{mg} / \mathrm{mL}^{*} \mathrm{~min}\right)\end{array}$ & 41 & 62 & 59 & 98 & 49 & 0 & High \\
\hline & $\begin{array}{l}\text { Paclitaxel }\left(200 \mathrm{mg} / \mathrm{m}^{2}\right)+\text { Carboplatin } \\
\left(6 \mathrm{mg} / \mathrm{mL} \mathrm{L}^{*} \mathrm{~min}\right)+\text { Bevacizumab } \\
(15 \mathrm{mg} / \mathrm{kg})\end{array}$ & 44 & 58 & 52 & 95 & 0 & 0 & \\
\hline \multirow[t]{2}{*}{ Spigel [35] } & $\begin{array}{l}\text { Ixabepilone }\left(30 \mathrm{mg} / \mathrm{m}^{2}\right)+\text { Carboplatin } \\
\left(6 \mathrm{mg} / \mathrm{mL}^{*} \mathrm{~min}\right)\end{array}$ & 42 & 63 & 57 & 69 & 47 & 0 & High \\
\hline & $\begin{array}{l}\text { Ixabepilone }\left(30 \mathrm{mg} / \mathrm{m}^{2}\right)+\text { Carbo- } \\
\text { platin }\left(6 \mathrm{mg} / \mathrm{mL}^{*} \mathrm{~min}\right)+\text { Bevaci- } \\
\text { zumab(15 mg/kg) }\end{array}$ & 40 & 63 & 48 & 67 & 3 & 0 & \\
\hline \multirow[t]{3}{*}{ Spigel [36] } & $\begin{array}{l}\text { Pemetrexed }\left(500 \mathrm{mg} / \mathrm{m}^{2}\right)+\text { Carbopl- } \\
\text { atin }\left(5 \mathrm{mg} / \mathrm{mL}^{*} \mathrm{~min}\right)+\text { Bevacizumab } \\
(15 \mathrm{mg} / \mathrm{kg})\end{array}$ & 61 & 73 & 56 & 97 & 0 & 100 & Moderate \\
\hline & Pemerexed $\left(500 \mathrm{mg} / \mathrm{m}^{2}\right)$ & 48 & 72 & 63 & 90 & 0 & 100 & \\
\hline & $\begin{array}{l}\text { Pemetrexed }\left(500 \mathrm{mg} / \mathrm{m}^{2}\right)+\text { Bevaci- } \\
\text { zumab }(15 \mathrm{mg} / \mathrm{kg})\end{array}$ & 63 & 72 & 57 & 92 & 0 & 100 & \\
\hline \multirow[t]{2}{*}{ Treat [37] } & $\begin{array}{l}\text { Gemcitabine }\left(1,000 \mathrm{mg} / \mathrm{m}^{2}\right)+\text { Carbo- } \\
\text { platin }\left(75 \mathrm{mg} / \mathrm{m}^{2}\right)\end{array}$ & 379 & 64.1 & 58.3 & 90 & 17.7 & 0.3 & Moderate \\
\hline & $\begin{array}{l}\text { Paclitaxel }\left(225 \mathrm{mg} / \mathrm{m}^{2}\right)+\text { Carboplatin } \\
\left(6 \mathrm{mg} / \mathrm{mL}^{*} \mathrm{~min}\right)\end{array}$ & 379 & 64.1 & 60.9 & 89.4 & 16.1 & 0.3 & \\
\hline \multirow[t]{2}{*}{ Wu [38] } & $\begin{array}{l}\text { Pemetrexed }\left(500 \mathrm{mg} / \mathrm{m}^{2}\right)+\text { Cisplatin } \\
\left(75 \mathrm{mg} / \mathrm{m}^{2}\right)\end{array}$ & 126 & 57 & 56.3 & 84.9 & 0 & 0 & High \\
\hline & $\begin{array}{l}\text { Gemcitabine }\left(1,250 \mathrm{mg} / \mathrm{m}^{2}\right)+\text { Cispl- } \\
\text { atin }\left(75 \mathrm{mg} / \mathrm{m}^{2}\right)\end{array}$ & 130 & 56 & 54.6 & 84.6 & 0 & 0 & \\
\hline \multirow[t]{2}{*}{ Zinner [39] } & $\begin{array}{l}\text { Pemetrexed }\left(500 \mathrm{mg} / \mathrm{m}^{2}\right)+\text { Carbopl- } \\
\text { atin }\left(6 \mathrm{mg} / \mathrm{mL}^{*} \mathrm{~min}\right)\end{array}$ & 182 & 66 & 57.5 & 99.5 & 0 & 0 & High \\
\hline & $\begin{array}{l}\text { Paclitaxel }\left(200 \mathrm{mg} / \mathrm{m}^{2}\right)+\text { Carboplatin } \\
\left(6 \mathrm{mg} / \mathrm{mL} \mathrm{L}^{*} \mathrm{~min}\right)+\text { Bevacizumab } \\
(15 \mathrm{mg} / \mathrm{kg})\end{array}$ & 179 & 65 & 58.1 & 100 & 0 & 0 & \\
\hline
\end{tabular}


Table 1 (continued)

\begin{tabular}{|c|c|c|c|c|c|c|c|c|}
\hline Study ID & Treatment & Sample size & Median age & Male (\%) & Stage IV (\%) & SCC (\%) & $\mathrm{PS}=2$ & Quality assesment \\
\hline \multirow[t]{2}{*}{ Zhao [40] } & $\begin{array}{l}\text { Endostar(7.5 mg/ } \mathrm{m}^{2} \text { on days } 1 \text { to } \\
\text { 14) + Gemcitabine (1000 mg/ } \\
\left.\mathrm{m}^{2} \text {,days } 1 \text { and } 8\right)+ \text { Cisplatin ( } \\
\left.30 \mathrm{mg} / \mathrm{m}^{2} \text {,day } 2-4\right)\end{array}$ & 33 & 61 & 63.4 & 84.85 & 45.45 & 9.09 & Moderate \\
\hline & $\begin{array}{l}\text { Gemcitabine }\left(1000 \mathrm{mg} / \mathrm{m}^{2} \text {,days } 1 \text { and }\right. \\
\left.\text { 8) + Cisplatin ( } 30 \mathrm{mg} / \mathrm{m}^{2} \text {, day } 2-4\right)\end{array}$ & 36 & 60 & 69.44 & 83.33 & 52.78 & 11.11 & \\
\hline \multirow[t]{2}{*}{ Zhou [41] } & $\begin{array}{l}\text { Paclitaxel }\left(175 \mathrm{mg} / \mathrm{m}^{2}\right)+\text { Carboplatin } \\
\left(6 \mathrm{mg} / \mathrm{mL}^{*} \mathrm{~min}\right)+\text { Bevacizumab } \\
(15 \mathrm{mg} / \mathrm{kg})\end{array}$ & 138 & 57 & 54.3 & 91.3 & 0 & 0 & High \\
\hline & $\begin{array}{l}\text { Paclitaxel }\left(175 \mathrm{mg} / \mathrm{m}^{2}\right)+\text { Carboplatin } \\
\left(6 \mathrm{mg} / \mathrm{mL}^{*} \mathrm{~min}\right)\end{array}$ & 138 & 56 & 55.8 & 90.6 & 0 & 0 & \\
\hline \multirow[t]{2}{*}{ Marinis [42] } & $\begin{array}{l}\text { Bevacizumab }(7.5 \mathrm{mg} / \mathrm{kg})+\text { Gemcit- } \\
\text { abine }\left(1,200 \mathrm{mg} / \mathrm{m}^{2}\right)\end{array}$ & 44 & 74.2 & 62.8 & 90.7 & 0 & 0 & High \\
\hline & $\begin{array}{l}\text { Bevacizumab }(7.5 \mathrm{mg} / \mathrm{k}) \mathrm{g}+\text { Cispl- } \\
\text { atin }\left(60 \mathrm{mg} / \mathrm{m}^{2}\right)+\text { Gemcitabine } \\
\left(1,000 \mathrm{mg} / \mathrm{m}^{2}\right)\end{array}$ & 42 & 73.9 & 70 & 97.5 & 0 & 0 & \\
\hline \multirow[t]{2}{*}{ Yu [43] } & $\begin{array}{l}\text { Gemcitabine }\left(1000 \mathrm{mg} / \mathrm{m}^{2}\right)+\text { Carbo- } \\
\text { platin }\left(5 \mathrm{mg} / \mathrm{mL}^{*} \mathrm{~min}\right)\end{array}$ & 25 & 56.7 & 76 & 60 & 56 & NA & High \\
\hline & $\begin{array}{l}\text { Gemcitabine }\left(1000 \mathrm{mg} / \mathrm{m}^{2}\right)+\text { Carbo- } \\
\text { platin }\left(5 \mathrm{mg} / \mathrm{mL}^{*} \mathrm{~min}\right)+\text { Endostar } \\
\left(7.5 \mathrm{mg} / \mathrm{m}^{2}\right)\end{array}$ & 24 & 56.3 & 70.8 & 62.5 & 50 & NA & \\
\hline \multirow[t]{2}{*}{ Schuette [44] } & $\begin{array}{l}\text { Bevacizumab }(7.5 \mathrm{mg} / \mathrm{kg})+\text { Pem- } \\
\text { etrexed }\left(500 \mathrm{mg} / \mathrm{m}^{2}\right)\end{array}$ & 119 & 72.3 & 62.2 & 95 & 0 & 5 & High \\
\hline & $\begin{array}{l}\text { Bevacizumab }(7.5 \mathrm{mg} / \mathrm{kg})+\text { Pem- } \\
\text { etrexed }\left(500 \mathrm{mg} / \mathrm{m}^{2}\right)+\text { Carboplatin } \\
\left(5 \mathrm{mg} / \mathrm{mL}^{*} \mathrm{~min}\right)\end{array}$ & 134 & 71.9 & 64.2 & 95.5 & 0 & 5 & \\
\hline \multirow[t]{2}{*}{ Zhou [45] } & $\begin{array}{l}\text { Pemetrexed }\left(500 \mathrm{mg} / \mathrm{m}^{2}\right)+\text { Cisplatin } \\
\left(75 \mathrm{mg} / \mathrm{m}^{2}\right)+\text { Endostar }\left(7.5 \mathrm{mg} / \mathrm{m}^{2}\right)\end{array}$ & 56 & 54.1 & 51.8 & 87.5 & 0 & 0 & Moderate \\
\hline & $\begin{array}{l}\text { Pemetrexed }\left(500 \mathrm{mg} / \mathrm{m}^{2}\right)+\text { Cisplatin } \\
\left(75 \mathrm{mg} / \mathrm{m}^{2}\right)\end{array}$ & 39 & 57.4 & 69.2 & 94.9 & 0 & 2.6 & \\
\hline \multirow[t]{2}{*}{ Liu [46] } & $\begin{array}{l}\text { Vinorelbine }\left(25 \mathrm{mg} / \mathrm{m}^{2}\right)+\text { Cisplatin } \\
\left(75 \mathrm{mg} / \mathrm{m}^{2}\right)+\text { Endostar }\left(7.5 \mathrm{mg} / \mathrm{m}^{2}\right)\end{array}$ & 19 & 55 & 57.9 & 57.0 & 36.8 & 5.3 & Moderate \\
\hline & $\begin{array}{l}\text { Vinorelbine }\left(25 \mathrm{mg} / \mathrm{m}^{2}\right)+\text { Cisplatin } \\
\left(75 \mathrm{mg} / \mathrm{m}^{2}\right)\end{array}$ & 34 & 58.4 & 52.9 & 61.8 & 20.6 & 11.8 & \\
\hline \multirow[t]{4}{*}{ Sun [47] } & $\begin{array}{l}\text { Endostar }\left(7.5 \mathrm{mg} / \mathrm{m}^{2} \text { on days } 1 \text { to }\right. \\
\begin{array}{l}\text { 14) + Vinorelbine }\left(25 \mathrm{mg} / \mathrm{m}^{2}\right)+\text { Cis- } \\
\text { platin }\left(75 \mathrm{mg} / \mathrm{m}^{2}\right)\end{array}\end{array}$ & 928 & 57.5 & 67.7 & 69.7 & 30.5 & NA & High \\
\hline & $\begin{array}{l}\text { Endostar }\left(7.5 \mathrm{mg} / \mathrm{m}^{2} \text { on days } 1 \text { to }\right. \\
\text { 14) + Paclitaxel }\left(150 \mathrm{mg} / \mathrm{m}^{2}\right)+\text { Cispl- } \\
\text { atin }\left(75 \mathrm{mg} / \mathrm{m}^{2}\right)\end{array}$ & 976 & 55.6 & 65.9 & 69.3 & 31.7 & NA & \\
\hline & $\begin{array}{l}\text { Endostar }\left(7.5 \mathrm{mg} / \mathrm{m}^{2} \text { on days } 1 \text { to }\right. \\
\text { 14) + Gemcitabine }(800 \sim 1000 \mathrm{mg} / \\
\left.\mathrm{m}^{2}\right)+ \text { Cisplatin }\left(75 \mathrm{mg} / \mathrm{m}^{2}\right)\end{array}$ & 441 & 58.1 & 75.3 & 71.7 & 33.1 & NA & \\
\hline & $\begin{array}{l}\text { Endostar }\left(7.5 \mathrm{mg} / \mathrm{m}^{2} \text { on days } 1 \text { to }\right. \\
14)+ \text { Gemcitabine (dose unavali- } \\
\text { able) + cisplatin (dose unavaliable) }\end{array}$ & 338 & 59.0 & 71.3 & 68.6 & 29.3 & NA & \\
\hline
\end{tabular}

between the network meta-analysis results and the head-to-head analysis results, we conducted nodesplit and heterogeneity analyses. For nodesplit analysis, in terms of ORR, the P values of Pla $+\mathrm{Pac}$ vs $\mathrm{Pla}+\mathrm{Gem}$ and Pla + Pem vs Pla + Gem were significantly different, at 0.0333 and 0.0450 , respectively, which indicated that the direct and indirect comparison results between $\mathrm{Pla}+\mathrm{Pac}$ vs $\mathrm{Pla}+\mathrm{Gem}$ and $\mathrm{Pla}+\mathrm{Pem}$ vs $\mathrm{Pla}+\mathrm{Gem}$ were inconsistent; for other comparisons of ORR and the nodesplit analysis results of OS and PFS, there were no significant differences. For the heterogeneity analysis, except for the high heterogeneity of Pla $+\mathrm{Pac}$ vs $\mathrm{Pla}+\mathrm{Pac}+\mathrm{Bev}(81.3 \%)$ for PFS, moderate heterogeneity of $\mathrm{Pla}+\mathrm{Pac}$ vs Pla $+\mathrm{Gem}$ and for ORR (65.1\%) and PFS (62.8\%), moderate heterogeneity of Pla + Pem vs $\mathrm{Pla}+\mathrm{Gem}(65.5 \%)$ and $\mathrm{Pla}+\mathrm{Pem}$ vs $\mathrm{Pla}+\mathrm{Pac}+\mathrm{Bev}$ 


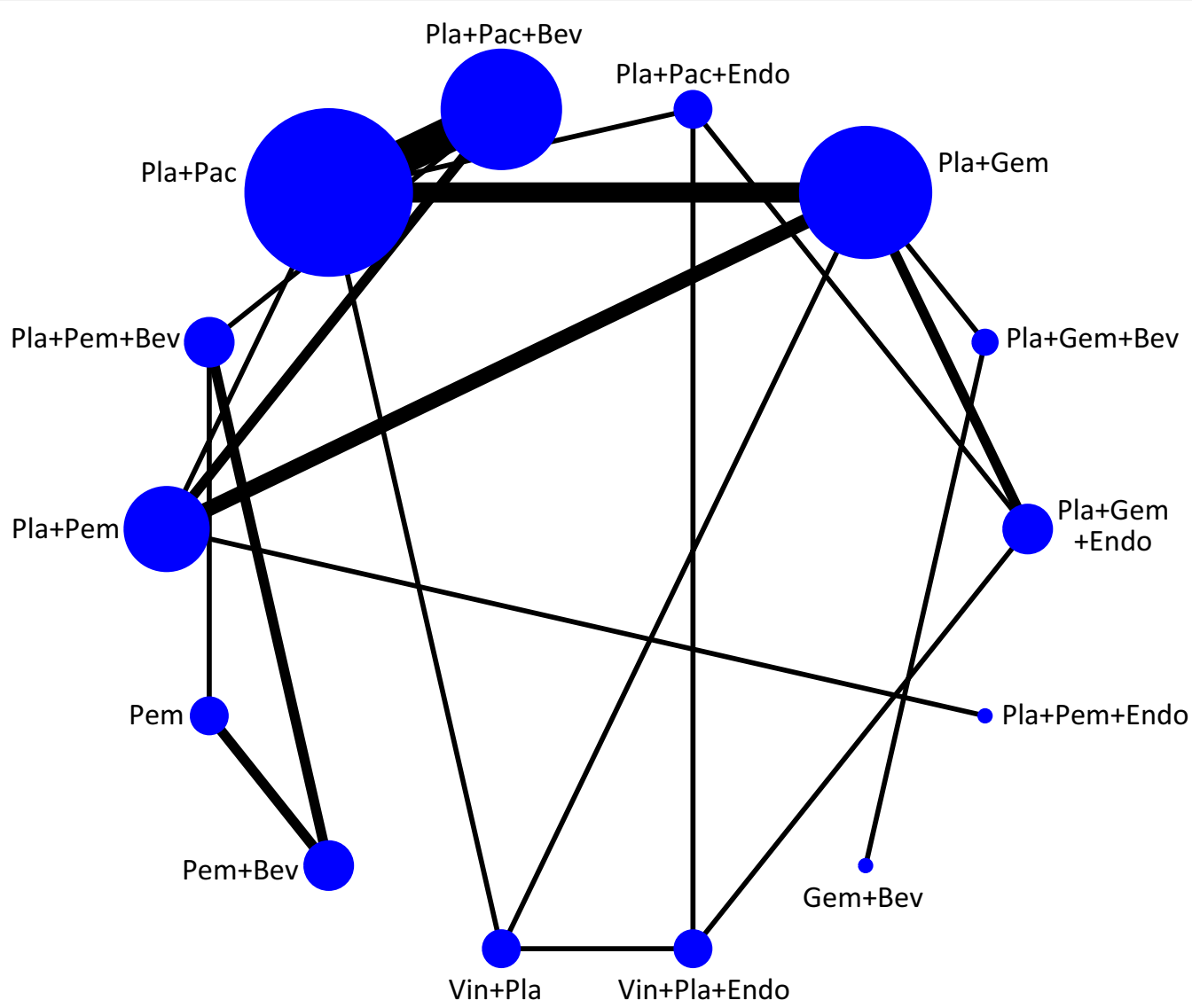

Fig. 2 Network plot of 14 interventions for the treatment of NSCLC according to Bayesian network meta-analysis. Each node represents a treatment, and the size of the node is proportional to the number of patients. The width of the lines between two nodes represents the number of head-to-head trials

(51.0\%) for ORR, there was small heterogeneity for the other results.

\section{Single-arm meta-analysis}

The forest plots of single-arm meta-analysis are shown in Fig. 6 (ORR) and Fig. 7 (OS and PFS). The forest plots of the event rate of adverse events are shown in Additional file 1: Figure S2.

\section{ORR}

The pooled ORR was 0.35 , and the $95 \%$ CI was $0.31,0.39$. Except for the synthesis result of Vin + Pla + Endo containing 0, the synthesis ORR values of $\mathrm{Pla}+\mathrm{Pac}+\mathrm{Bev}, \mathrm{Pla}+\mathrm{Pem}+\mathrm{Bev}$, were similar, which were 0.44 (0.37, 0.52), 0.40 (0.32, 0.48); followed by $\mathrm{Pem}+\mathrm{Bev}$ and $\mathrm{Pla}+\mathrm{Gem}+\mathrm{Bev}$ and $\mathrm{Pla}+\mathrm{Gem}+$ Endo, which were 0.35 (0.24, 0.46), 0.34 $(0.30,0.39)$ and $0.32(0.18,0.46)$, respectively. And followed by $\mathrm{Pla}+\mathrm{Pac}+$ Endo and $\mathrm{Pla}+\mathrm{Pem}+$ Endo, with synthesis ORR values of $0.27(0.06,0.49)$ and
$0.22(0.20,0.25)$, respectively. Gem $+\mathrm{Bev}$ was the worst intervention among them, with pooled ORR: $0.18(0.07,0.30)$.

2. OS and PFS

The pooled OS was 15.02 months, and the $95 \%$ CI was $12.70,17.34$. In addition to the result of $\mathrm{Gem}+\mathrm{Bev}$ containing $0, \mathrm{Pla}+\mathrm{Pem}+$ Endo had the largest synthesis result: 36.00 months with a $95 \%$ CI of 12.70 , 17.34; the second largest was that of $\mathrm{Pla}+\mathrm{Pac}+\mathrm{Bev}$ : 19.54 months with a 95\% CI of 12.19, 26.88; followed by Vin + Pla + Endo, $\mathrm{Pla}+\mathrm{Pac}+$ Endo and $\mathrm{Pla}+\mathrm{Gem}+$ Endo, with synthesized values of 16.96 (15.29, 18.60), 15.41 (12.36, 18.46) and 14.23(6.92, 21.53), respectively; the worst synthesis result was $\mathrm{Pla}+\mathrm{Gem}+\mathrm{Bev}$, with a result of 8.20 (3.40, 13.00). The pooled HR of OS was 0.89 (0.81, 0.98). The comparison between the included Endostar combined with chemotherapy regimens with chemotherapy regimens $(\mathrm{Pla}+\mathrm{Gem}+$ Endo vs $\mathrm{Pla}+\mathrm{Gem}$, $\mathrm{Pla}+$ Pem + Endo vs Pla + Pem) had no statistical significance, with HR value and 95\% CI 1.03(0.86, 1.23) 


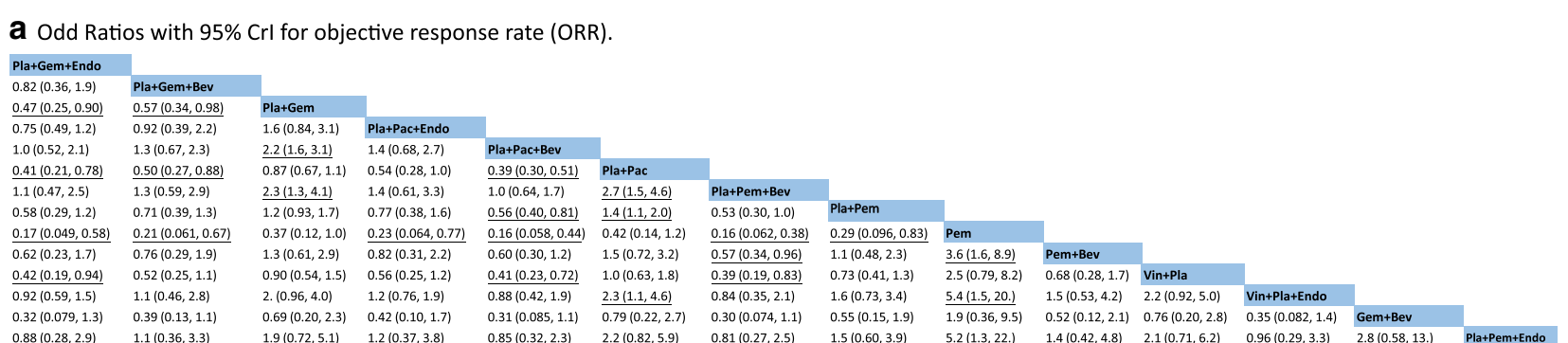

b Hazard Ratios with $95 \%$ Crl for overall survival (OS).

PlatGem+Endo
$13(0.67 .25)$
$12.62,23$

$1.3(0.67,2.5) \quad \mathrm{Pla}+\mathrm{Gem}+\mathrm{Be}$

$1.2(0.91,1.5) \quad 0.90(0.48,1.7)$

$1.0(0.56,1.9) \quad 0.81(0.62,1.1)$

$1.0(0.56,19)-0.81(0.62,1,1)$

$1.3(0.70,2.4) \quad 1.0(0.78,1.3)$

$1.1(0.58,2.0) \quad 0.84(0.66,1.1)$

$1.2(0.40,3.7) \quad 0.94(0.37,2.5)$
0

$0.95(0.45,2.0) \quad 0.75(0.46,1.2)$

$1.5(0.77,2.9) \quad 1.2(0.84,1.6)$

$\begin{array}{ll}1.0(0.80,1.3) & 0.80(0.41,1.5) \\ 0.91(0.18,4.2) & 0.70(0.16,3.0)\end{array}$

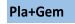

$0.92(0.52,1.6) \quad$ Pla+PactEndo

$0.83(0.71,0.97) \quad 0.90(0.51,1.6)$

$\frac{0.83(0.71,0.97)}{1.0(0.93,1.1)} \quad 1.1(0.64,2$.

$0.83(0.65,1.1) \quad 0.90(0.49,1.7)$

$0.87(0.77,0.99) \quad 0.94(0.53,1.7)$

$0.97(0.39,2.5) \quad 1.0(0.36,3.1)$

$0.77(0.50,1.2) \quad 0.83(0.41,1.7)$

$1.2(0.95,1.5) \quad 1.3(0.70,2.4)$

$0.82(0.44,1.5) \quad 0.89(0.70,1.1)$

\section{Pla+Pac+Bev}

$\frac{1.2(1.1,1.4)}{10(0.82,1.2)} \quad$ Pla+Pac

$0.81(0.64,1.0) \quad$ Pla+Pem+Bev

$0.85(0.74,0.98) \quad 1.0(0.81,1.4)$

$\begin{array}{lll}1.2(0.47,3 .) & 0.94(0.38,2.4) & 1.2(0.48,2.9)\end{array}$

$\begin{array}{lll}0.92(0.61,1.4) & 0.75(0.49,1.1) & 0.92(0.65,1.3) \\ 1.4(1.1,1.8) & 1.2(0.93,1.4) & 1.4(1.0,2 .)\end{array}$

$\begin{array}{lll}1.4(1.1,1.8) & 1.2(0.93,1.4) & 1.4(1.0,2 .) \\ 0.99(0.53,1.8) & 0.80(0.43,1.5) & 0.99(0.51,1.9) \\ 0.870 .20 .3) & 0.70(0.16) & 0.870 .30 .7)\end{array}$

$1.1(0.44,2.9) \quad P$ Pe

$0.88(0.57,1.4) \quad 0.79(0.34,1.8) \quad$ Pem+Bev

$\begin{array}{llll}1.4(1.1,1.7) & 1.2(0.47,3.1) & 1.5(0.96,2.5) & \text { Vin }+ \text { Pla }\end{array}$

$0.85(0.28,2.5) \quad 1.1(0.51,2.3) \quad 0.69(0.36,1.3) \quad$ VintPla+Endo

$\begin{array}{llllllll}0.87(0.20,3.6) & 0.70(0.16,3 .) & 0.87(0.20,3.7) & 0.83(0.19,3.5) & 0.74(0.13,4.1) & 0.94(0.21,4.2) & 0.61(0.14,2.6) & 0.89(0.18,4.1)\end{array}$

C Hazard Ratios with $95 \%$ Crl for progression free survival (PFS).

\begin{tabular}{|c|c|c|c|}
\hline Pla+Gem+Endo & & & \\
\hline $1.3(0.59,2.9)$ & $\mathrm{Pla}+\mathrm{Gem}+\mathrm{Bev}$ & & \\
\hline $1.6(0.85,3.0)$ & $1.2(0.76,2)$. & $\mathrm{Pla}+\mathrm{Gem}$ & \\
\hline $1.6(0.65,3.8)$ & $1.2(0.54,2.6)$ & $0.97(0.52,1.8)$ & Pla + PactEndo \\
\hline $0.92(0.45,1.9)$ & $0.70(0.40,1.2)$ & $0.57(0.42,0.79)$ & $0.59(0.31,1.1)$ \\
\hline $1.8(0.90,3.5)$ & $1.3(0.80,2.3)$ & $1.1(0.88,1.4)$ & $1.1(0.63,2.0)$ \\
\hline $1.1(0.48,2.6)$ & $0.84(0.40,1.8)$ & $0.69(0.40,1.2)$ & $0.71(0.32,1.6)$ \\
\hline $1.5(0.74,3)$. & $1.1(0.65,2)$. & $0.93(0.70,1.2)$ & $0.95(0.49,1.8)$ \\
\hline $1.7(0.44,6.6)$ & $1.3(0.36,4.7)$ & $1.1(0.32,3.5)$ & $1.1(0.30,4.0)$ \\
\hline $1.4(0.53,3.9)$ & $1.1(0.44,2.7)$ & $0.89(0.42,1.9)$ & $0.92(0.36,2.3)$ \\
\hline $1.8(0.84,3.9)$ & $1.4(0.72,2.6)$ & $1.1(0.73,1.8)$ & $1.2(0.56,2.4)$ \\
\hline $1.3(0.51,3.4)$ & $0.99(0.42,2.3)$ & $0.81(0.40,1.7)$ & $0.84(0.33,2.1)$ \\
\hline $1.2(0.52,2.9)$ & $0.92(0.43,2)$. & $0.76(0.42,1.4)$ & $0.78(0.34,1.8)$ \\
\hline
\end{tabular}

Fig. 3 ORs or HRs between the included interventions according to the results of network meta-analysis (the treatment in the column compared with the treatment in the row)

and $0.81(0.18,3.60)$, respectively; Among the bevacizumab combined with chemotherapy regimens vs chemotherapy regimens, only $\mathrm{Pla}+\mathrm{Pac}+\mathrm{Bev}$ vs $\mathrm{Pla}+\mathrm{Pac}$ had statistical significance: 0.78 (0.69, 0.89); For PFS, the pooled HR and 95\% CI were 0.67 (0.56, 0.81). Only Pla + Gem + Endo vs Pla + Gem was statistically siginificant among the results of comparison of Endostar combined with chemotherapy regimens with chemotherapy regimens, with HR value and 95\% CI $0.62(0.39,0.99)$. For bevacizumab combined with chemotherapy vs chemotherapy regimens, $\mathrm{Pla}+\mathrm{Pac}+\mathrm{Bev}$ vs $\mathrm{Pla}+\mathrm{Pac}$ and $\mathrm{Pla}+\mathrm{Gem}+\mathrm{Bev}$ vs Pla + Gem could significantly prolong PFS, with HR values of $0.51(0.38,0.67)$ and $0.82(0.68,0.98)$, respectively.

3. Event rate of adverse events

Four most common adverse events between bevacizumab combined with chemotherapy and Endostar combined with chemotherapy, including anemia, thrombocytopenia, leukopenia and vomiting, were analysed in the current study. For anemia, the pooled event rate was $0.42(0.29,0.54)$. Except the result of $\mathrm{Pla}+\mathrm{Gem}+$ Endo containing 0, the interventions with the highest incidence of anemia were Pem + Bev $[0.58(0.21,0.96)]$ and $\mathrm{Pla}+\mathrm{Pem}+\mathrm{Bev}$ $[0.55(0.36,0.74)]$, followed by $\mathrm{Pla}+\mathrm{Pac}+\mathrm{Bev}[0.32$ $(0.08,0.57)]$ and Pla + Gem + Bev $[0.30(0.16,0.44)]$, and the lowest was Gem + Bev [0.09 (0.01, 0.18)]. The pooled event rate of leukopenia was 0.57 (0.32, 0.82). Expect the result of $\mathrm{Gem}+\mathrm{Bev}$ containing 0 , the highest three were: $\mathrm{Pla}+\mathrm{Pac}+$ Endo $[0.87$ $(0.79,0.95)], \mathrm{Pla}+\mathrm{Pac}+\mathrm{Bev}[0.76(0.39,1.13)]$ and $\mathrm{Pla}+$ Gem + Endo [0.68 (0.27, 1.09)]; the intervention with the lowest incidence of leukopenia was $\mathrm{Pla}+\mathrm{Gem}+\mathrm{Bev}[0.28(0.14,0.41)]$. For thrombocytopenia, the pooled event rate was $0.30(0.15$, 0.44). The intervention with the highest incidence of thrombocytopenia was $\mathrm{Pla}+\mathrm{Pem}+\mathrm{Bev}[0.48$ (0.39, 0.57)]; the lowest was Pla + Pem + Endo [0.14 (0.05, $0.23)]$ and Gem $+\operatorname{Bev}[0.12(0.02,0.21)]$. Finally, the 
a Heat map of the rank probabilities of objective response rate (ORR).

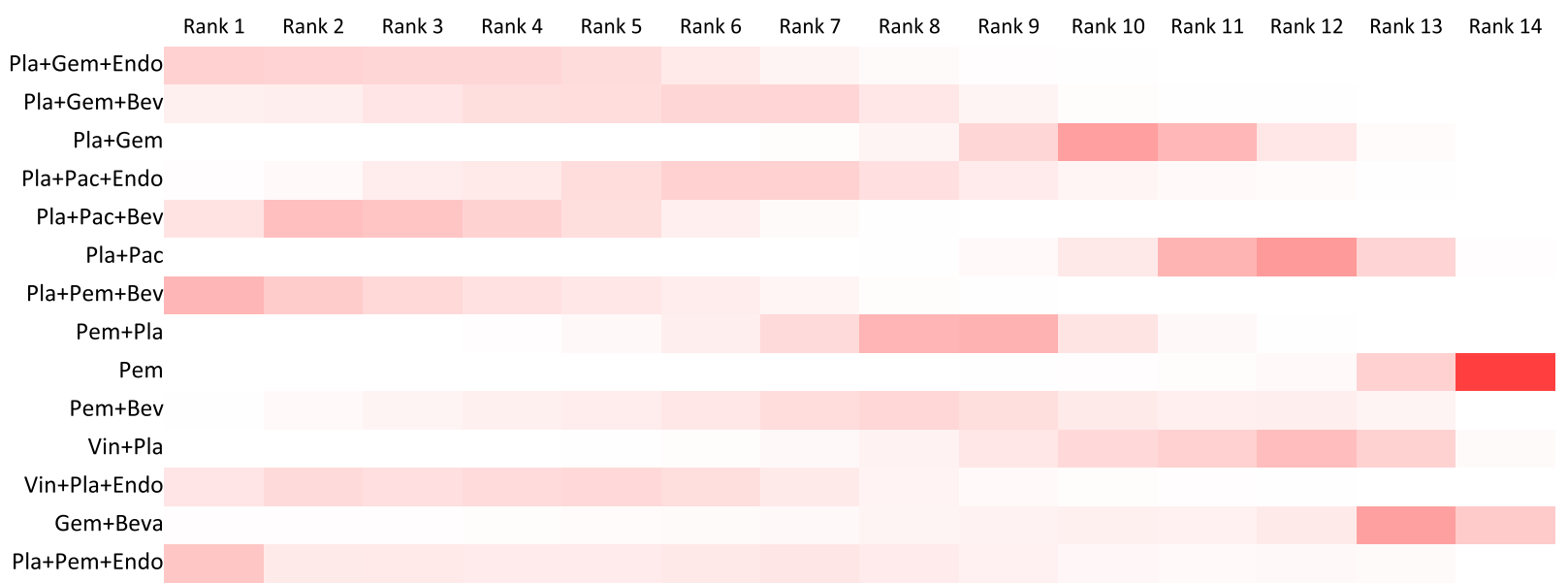

b Heat map of the rank probabilities of overall survival (OS).

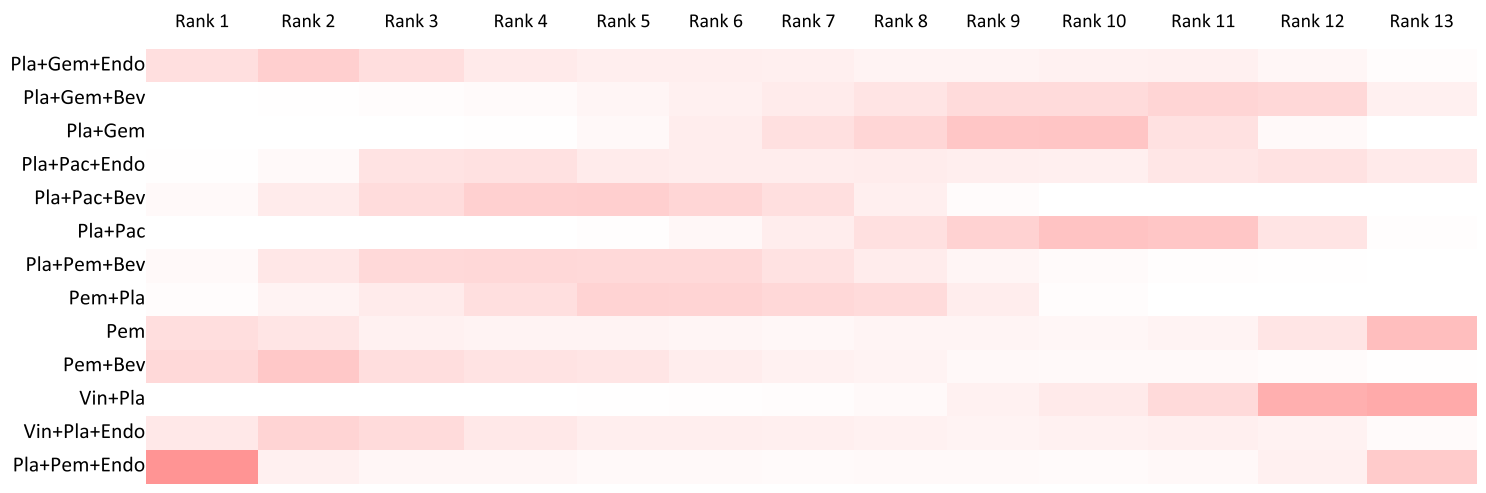

c Heat map of the rank probabilities of progression free survival (PFS).

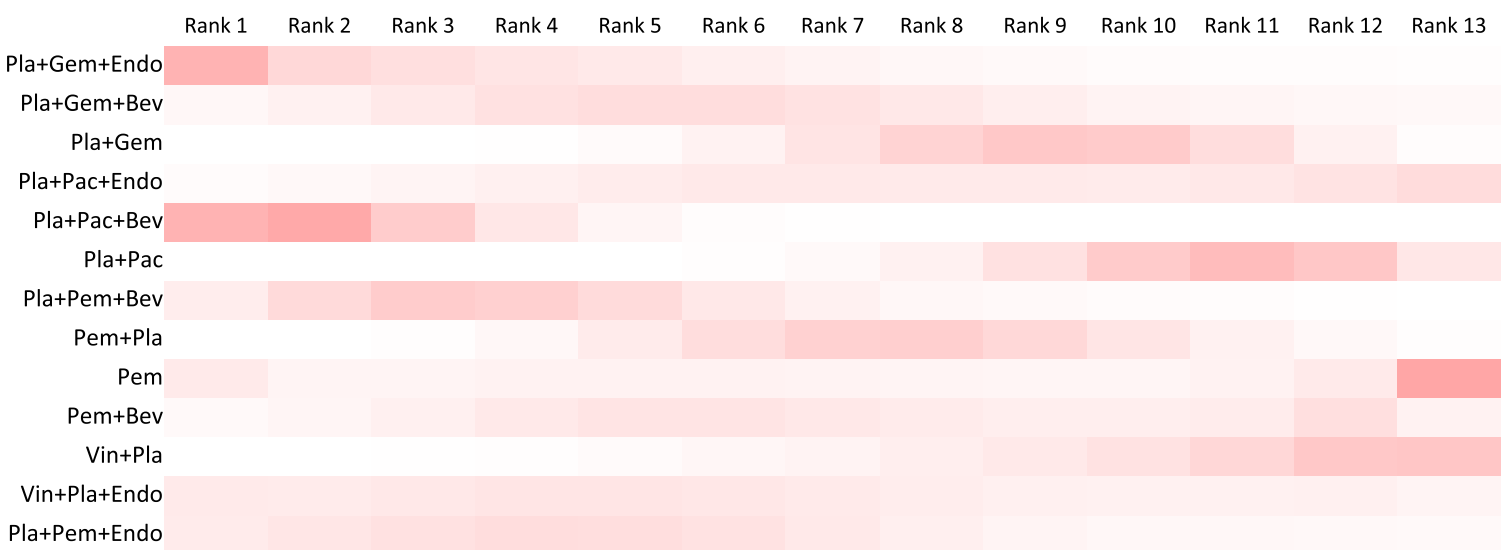

\section{0}

Fig. 4 Heat map of the rank probabilities of each intervention according to the results of the network meta-analysis. Figure 3a: ORR. Figure 3b: OS. Figure 3c: PFS. The detailed data are provided in Additional file 1: Table S1 
$a_{1}$

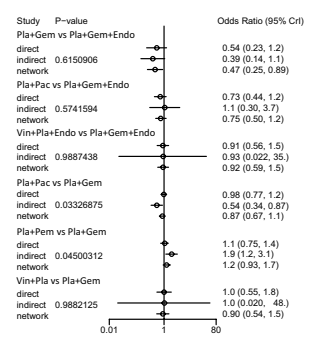

b

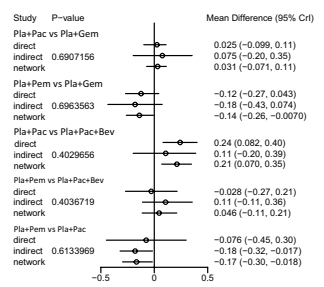

$\mathbf{d}_{3}$

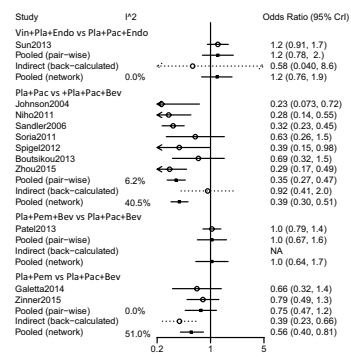

$\mathbf{e}_{1}$

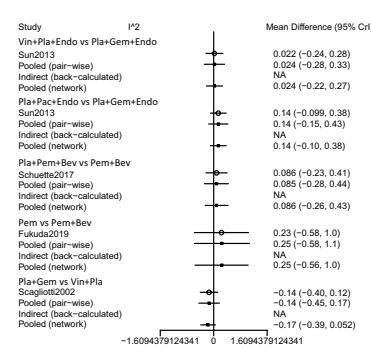

$\mathbf{a}_{2}$

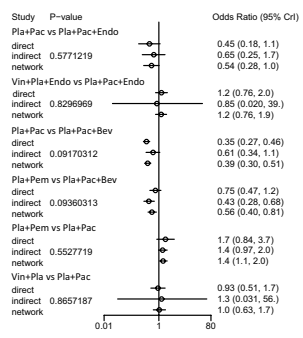

C

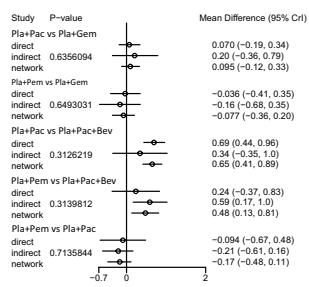

$\mathrm{d}_{4}$

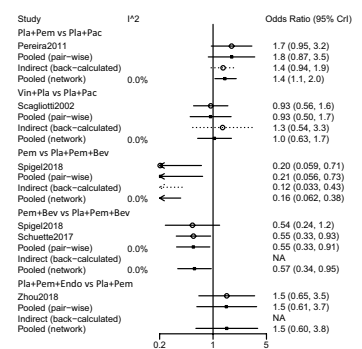

$\mathbf{e}_{2}$

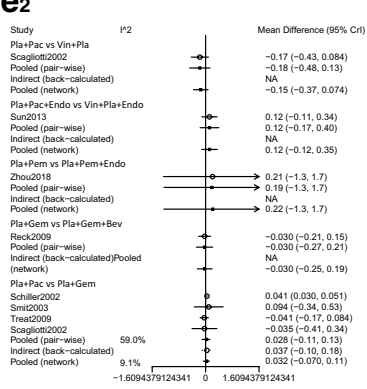

$a_{3}$

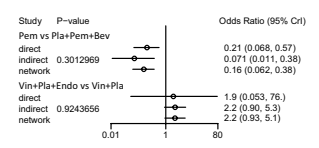

$\mathbf{d}_{1}$

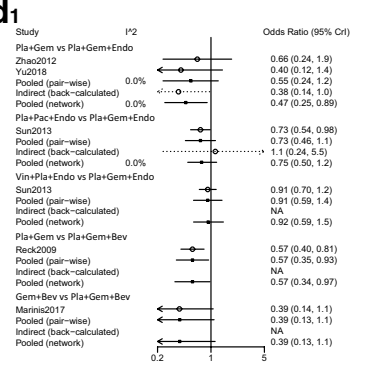

$d_{5}$

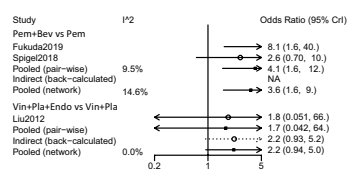

$\mathbf{e}_{3}$

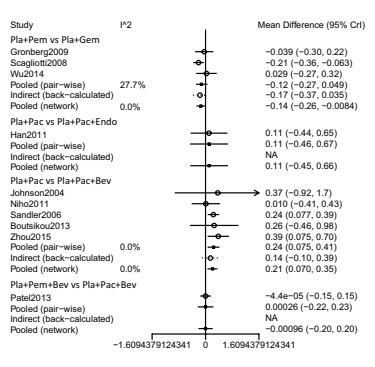

$\mathrm{d}_{2}$

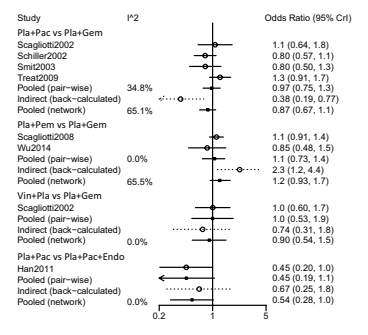

f1

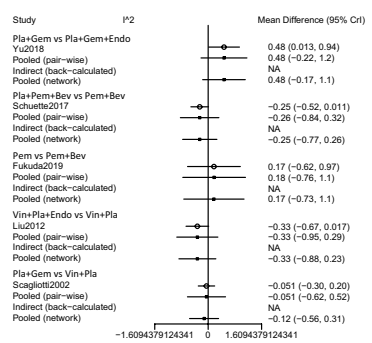

$f_{2}$

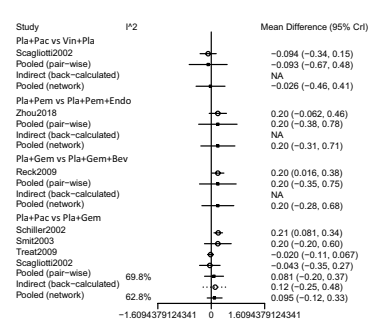

$\mathbf{f}_{3}$

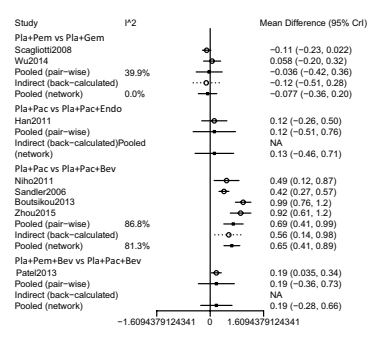

$\mathbf{e}_{4}$

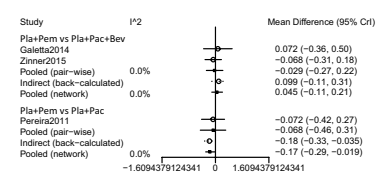

Fig. 5 Nodesplit analysis and analysis of heterogeneity for ORR, OS and PFS. a1 $\sim \mathbf{a}_{\mathbf{3}}$ Nodesplit analysis of ORR. b Nodesplit analysis of OS. C: Nodesplit analysis of PFS. $\mathbf{d} \mathbf{1} \sim \mathbf{d}_{\mathbf{5}}$ Analysis of heterogeneity for ORR. $\mathbf{e} \mathbf{1} \sim \mathbf{e}_{\mathbf{4}}$ Analysis of heterogeneity for OS. $\mathbf{f} \mathbf{1} \sim \mathbf{f}_{\mathbf{4}}$ Analysis of heterogeneity for PFS 


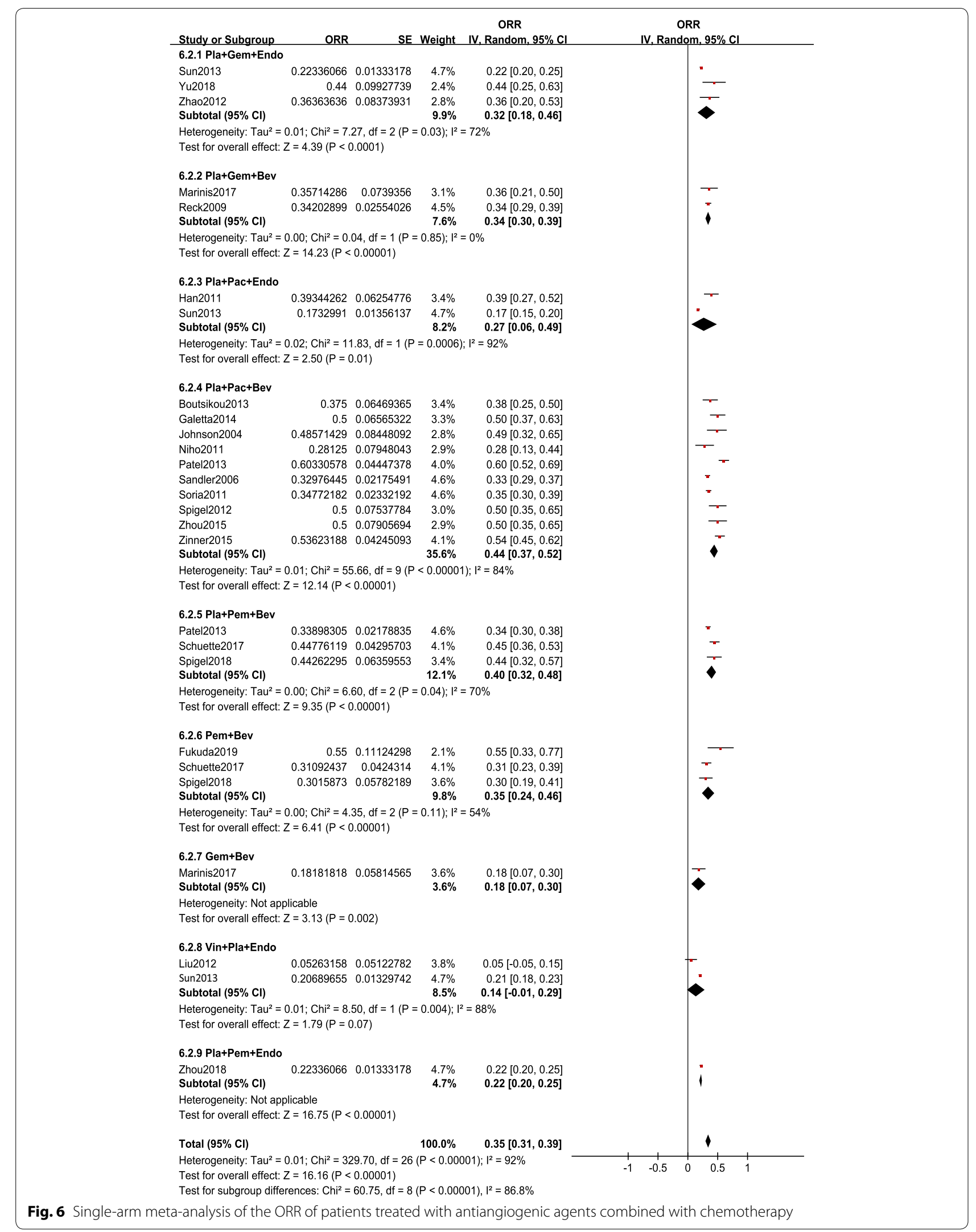


a Results of single-arm meta-analysis for OS.

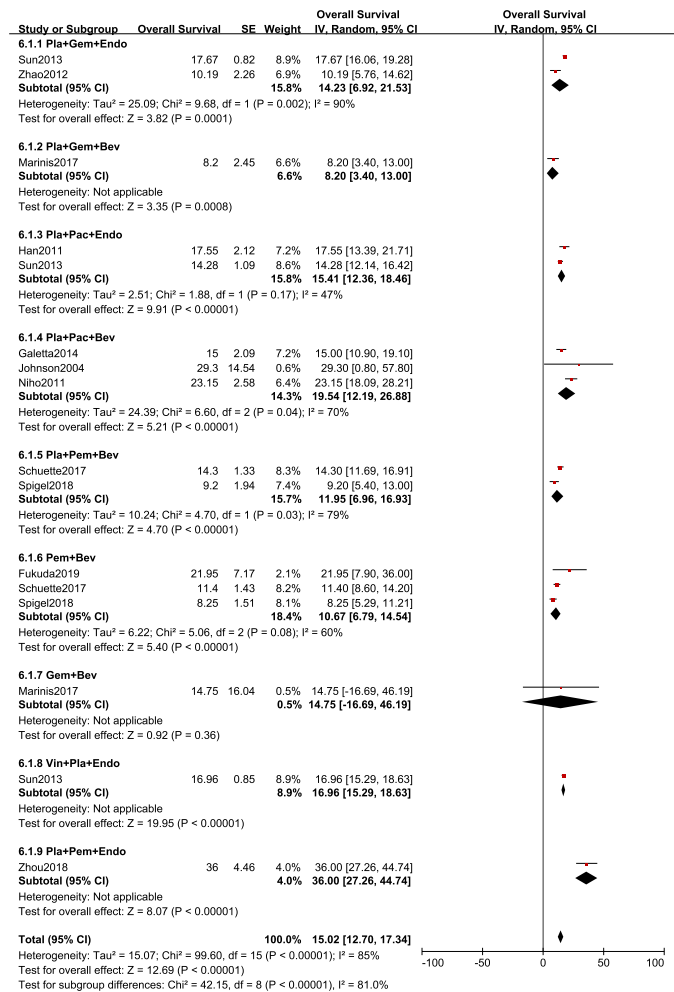

b Results of single-arm meta-analysis for PFS.

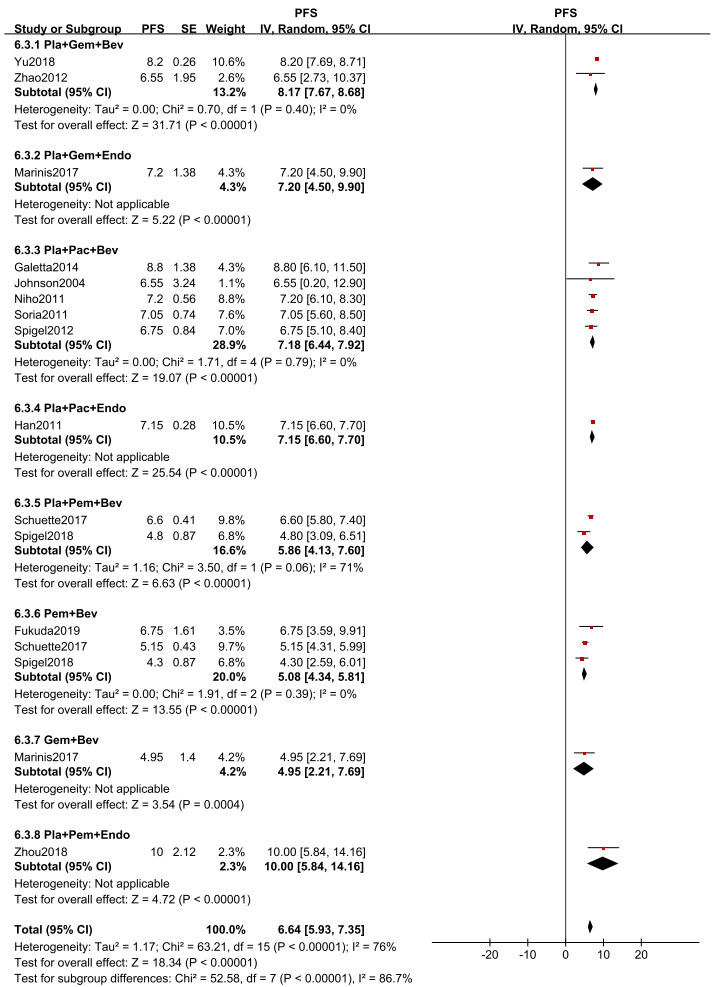

c Results of single-arm meta-analysis for HRs of OS.

d Results of single-arm meta-analysis for HRs of PFS.
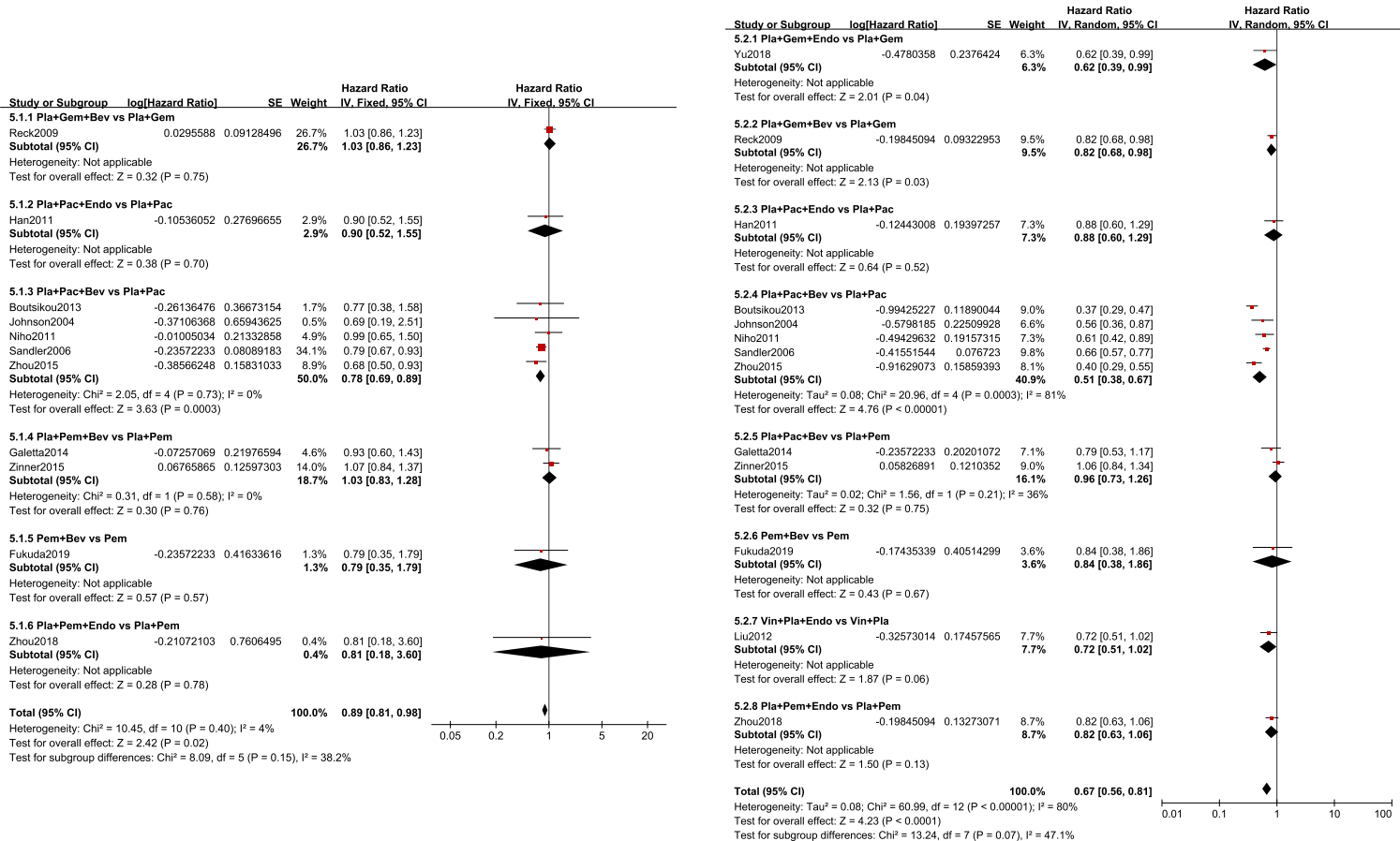

Fig. 7 Single-arm meta-analysis of the OS and PFS of patients treated with antiangiogenic agents combined with chemotherapy 
pooled event rate of vomiting was $0.20(0.11,0.30)$. In addition to the result of Gem + Bev containing 0 , $\mathrm{Pla}+$ Pem + Endo had the highest incidence [0.86 $(0.77,0.95)]$, while Pla + Pac + Endo $[0.15(0.06,0.23)]$ and $\mathrm{Pla}+\mathrm{Gem}+\mathrm{Bev}[0.09(0.06,0.12)]$ had the lowest incidence of vomiting. For leukopenia and thrombocytopenia, potential publication bias may exist according to the results of Eegger's test $(p=0.006$ and $p=0.002$, respectively). More details of the results of Begg's and Egger's test and funnel plots are shown in Additional file 1: Table S2 and Figure S3.

\section{Discussion}

Angiogenesis plays an important role in tumorigenesis and development and is related to tumor proliferation, invasion and metastasis [4]. The results of clinical studies has suggested the combination of antiangiogenic agents and chemotherapy was effective, which can improve the ORR and prolong the OS and PFS for NSCLC patients. However, bevacizumab combined with chemotherapy and Endostar combined with chemotherapy are still lack of head-to-head clinical trials, which limits the clinical application of antiangiogenic agents combined with chemotherapy in the treatment of NSCLC. Therefore, by conducting the current Bayesian network meta-analysis, the effects of 14 interventions included in our study on improving ORR, prolonging PFS and OS were compared indirectly, so as to provide possible suggestions for further clinical trials and rational application of antiangiogenic agents and chemotherapy in the treatment of NSCLC. Our study showed that antiangiogenic agents combined with dual chemotherapy agents containing platinum may have a better effect on NSCLC patients. In terms of ORR, bevacizumab combined with chemotheray may have a better effect for the treatment of NSCLC; and in terms of OS and PFS, Endostar combined with chemotherapy may be superior to bevacizumab combined with chemotherapy.

The results of the single-arm meta-analysis showed that the combination of antiangiogenic agents and chemotherapy agents could improve ORR [pooled ORR and 95\% CI: $0.35(0.31,0.39)]$, OS [pooled HR and 95\% CI: 0.89 $(0.81,0.98)$ ] and PFS [pooled HR and 95\% CI: 0.67 (0.56, $0.81)$, which showed that antiangiogenic agents combined with chemotherapy was more effective than chemotherapy alone. However, at present, it is still unclear that antiangiogenic agents in combination with which chemotherapy regimen can provide more benefits to patients in the treatment of NSCLC. Based on the first-ranked and second-ranked interventions of rank probabilities, our analysis found that, in terms of ORR, antiangiogenic agents combined with $\mathrm{Pla}+\mathrm{Pem}$ or $\mathrm{Pla}+\mathrm{Pac}$ may have better therapeutic effects; in terms of OS, antiangiogenic agents combined with Pla + Pem or Pem may be superior to prolonging $\mathrm{OS}$ and in terms of PFS, combined with $\mathrm{Pla}+\mathrm{Gem}$ or $\mathrm{Pla}+\mathrm{Pac}$ may have better effects. Therefore, our results showed than antiangiogenic agents combined with Pla + Pem, Pla + Pac or Pla + Pem may benefit patients more in the treatment of NSCLC. Among them, antiangiogenic agents combined with $\mathrm{Pla}+\mathrm{Pem}$ seems to be more conducive to improving ORR and prolonging OS and combined with Pla + Pac seems to be the first choice for prolonging $\mathrm{OS}$ and PFS.

The combinations of Endostar, an antiangiogenic agent, combined with chemotherapy and bevacizumab combined with chemotherapy have shown good results. However, the comparison between Endostar combined with chemotherapy and bevacizumab combined with chemotherapy lacks head-to-head clinical trials. Our results suggested that there was no significant difference in the effect of improving ORR and prolonging OS and PFS in NSCLC patients between Endostar combined with chemotherapy and bevacizumab combined with chemotherapy. Although there were no significant differences between these interventions, the rank positions indicated that $\mathrm{Pla}+\mathrm{Pem}+\mathrm{Bev}$ and $\mathrm{Pla}+\mathrm{Pac}+\mathrm{Bev}$ were in a more advanced position for improving ORR, and in terms of OS, Pla + Gem + Endo may be the best choice among these interventions. And interesting, for prolnging PFS, our results showed that Pla + Pem + Endo may be the first-ranked regimen, however, as the only bevacizumab combined with chemotherapy regimen for the treatment of non-squamous NSCLC aproved by FDA, $\mathrm{Pla}+\mathrm{Pac}+\mathrm{Bev}$ was the second-ranked regimen for improving PFS. Our analysis indicaded that bevacizumab combined with chemotherapy seems more effective for improving ORR and Endostar seems more effective for prolonging OS and PFS.

For the comparison of the different Endostar combined with chemotherapy regimens, a 4-phase clinical study showed that there were no significant differences in improving the ORR, OS and TTP (time to progression) among Vin $+\mathrm{Pla}+$ Endo, $\mathrm{Pla}+\mathrm{Gem}+$ Endo and $\mathrm{Pla}+\mathrm{Pac}+$ Endo [47]. However, for $\mathrm{Pla}+\mathrm{Pem}+$ Endo, there was few head-to-head comparisons with other Endostar combined chemotherapy regimens. Our network meta-analysis showed that compared with Vin $+\mathrm{Pla}+$ Endo, Pla $+\mathrm{Gem}+$ Endo and $\mathrm{Pla}+\mathrm{Pac}+$ Endo, there were no statistical difference in improving ORR and prolonging OS and PFS. However, the results of single-arm meta-analysis showed that some differences still existed among them. Compared with $\mathrm{Pla}+\mathrm{Gem}+$ Endo, the ORR [ $0.22(0.20,0.25)$ vs $0.32(0.18,0.46)]$, OS [ 36.00 months $(27.26,44.74)$ vs 14.23 months $(6.92,21.23)]$ and PFS [10.00 months (5.84, 
14.16) vs 8.17 months $(7.67,8.68)]$ of $\mathrm{Pla}+\mathrm{Pem}+$ Endo were obviously lower or longer. Similarly, compared with $\mathrm{Pla}+\mathrm{Pac}+$ Endo, the OS [ 36.00 months $(27.26,44.74)$ vs 15.41 months $(12.36,18.46)]$ and PFS [10.00 months $(5.84,14.16)$ vs 7.15 months $(6.60,7.70)]$ of $\mathrm{Pla}+\mathrm{Pem}+$ Endo were evidently longer, although there was no obvious difference for ORR [ $0.22(0.20,0.25)$ vs $0.27(0.06,0.49)]$. The reason may be related to the differences and inconsistencies between the studies and the limited number of studies. In the future, head-to-head randomized controlled trials are needed to determine the relationship between the above strategies for improving the prognosis of patients to provide more reasonable treatments for NSCLC patients.

For adverse events, the event rate of leukopenia [0.57 $(0.32,0.82)]$ was the highest among the four most common adverse events between Endostar combined with chemotherapy and bevacizumab combined with chemotherapy. It is worth noting that the common adverse events of bevacizumab combined with chemotherapy, such as hypertension, proteinuria and thromboembolism, have not been studied in the study of Endostar combined with chemotherapy. Compared with bevacizumab combined with chemotherapy, in $\mathrm{Pla}+\mathrm{Pac}+$ Endo, the event rate of leukopenia was the highest $[0.87(0.79,0.95)]$, while the incidence of vomiting was the second lowest $[0.15(0.06,0.23)]$. In addition, in Pla + Pem + Endo, the event rate of vomiting was the highest $[0.86(0.77,0.95)]$ and the incidence of thrombocytopenia was the second lowest $[0.14(0.05,0.23)]$. Gem + Bev had a low incidence in both anemia $[0.09(0.01,0.18)]$ and thrombocytopenia $[0.12(0.02,0.21)]$. However, due to the high heterogeneity, limited number and potential publication bias of studies, further studies are needed to confirm these conclusions.

For our network meta-analysis, there were still some limitations. First, we included 11 moderate-quality studies and 18 high-quality studies: of the 25 randomized controlled trials, 10 were moderate-quality articles and 15 were high-quality articles; the other 4 were nonrandomized controlled trials and prospective studies, of which 3 were high-quality articles and 1 was a moderate quality article according to the NOS. Although the number of included nonrandomized controlled trials and prospective studies was relatively small, the methodological design of the randomized clinical trials was still more reliable than that of the nonrandomized controlled trials, which may lead to inconsistency in our results. Second, for the patients who participated in the study, squamous cell carcinoma and non-squamous cell carcinoma were not distinguished, which may have some impact on the consistency of our results. For squamous cell carcinoma, there were no independent study data and thus, we failed to observe the curative effect of Endostar combined with chemotherapy and bevacizumab combined with chemotherapy in squamous cell carcinoma. Third, in order to facilitate the analysis, we did not make a strict distinction between the dosage and the method of administration. In addition, original data and laboratory data was lacking in the current meta-analysis. Finally, our results were based on a Bayesian network meta-analysis and the statistical analysis of various interventions; however, the performance of various interventions in real patients still needs to be confirmed by head-to-head clinical trials.

\section{Conclusions}

In summary, the combination of antiangiogenic agents with platinum-containing dual drug chemotherapy can improve NSCLC patients' benefit. The combination of Endostar and platinum-containing dual drugs may be a better choice to prolong OS and PFS. More clincial trials are needed to ensure the reasonable use of antiangiogenic combined with chemotheray regimens for NSCLC patients in the future.

\section{Supplementary information}

Supplementary information accompanies this paper at https://doi. org/10.1186/s12935-020-01639-4.

Additional file 1: Table S1. Rank probabilities of each treatment for different outcome measures based on the network meta-analysis. Table S2. Begg's and Egger's tests of the single-arm meta-analysis. Table S3. PRISMA checklist of the current network meta-analysis. Figure S1. The details of quality assessment of included studies. A. The details of quality assessment using the Cochrane Collaboration's risk of bias tool for 25 randomized controlled trials. B. The details of quality assessment using Newcastle Ottawa Scale for 4 nonrandomized controlled trials. Figure S2. Results of single-arm meta-analysis of four common adverse events of bevacizumab combined with chemotherapy and Endostar combined with hemotherapy. A. Anemia; B. Leukopenia. C. Thrombocytopenia. D. Vomiting. Figure S3. Funnel plots of the single-arm meta-analyisis. A: ORR. B: OS. C: PFS. D: HR of OS. E: HR of PFS. F: Anemia. G: Leukopenia. H: Thrombocytopenia. I: Vomiting.

\section{Abbreviations}

NSCLC: Non-small cell lung cancer; VEGF: Vascular endothelial growth factor; PDGF: Platelet-derived growth factor; TGF- $\beta$ : Transforming growth factor- $\beta$; FGF: Fibroblast growth factor; ORR: Objective response rate; PFS: Progressionfree survival; OS: Overall survival; TTP: Time to progression; RCT: Randomized controlled trial; NOS: Newcastle ottawa scale; OR: Odd ratio; HR: Hazard ratio; Cl: Confidence intervals; Crl: Credible intervals; Pla + Gem + Endo: Platinum + gemcitabine + Endostar; Pla + Gem + Bev: Platinum + gemcitabine + bevaczumab; Pla + Gem: Platinum + gemcitabine; Pla + Pac + Endo: Platinum + paclitaxel + Endostar; $\mathrm{Pla}+\mathrm{Pac}+\mathrm{Bev}$ : Platinum + paclitaxel + bevacizumab; Pla + Pac: Platinum + paclitaxel; Pla + Pem + Bev: Platinum + pemetrexed + bevacizumab; Pla + Pem: Platinum + pemetrexed; Pem + Bev: Pemetrexed + bevacizumab; Vin + Pla: Vinorelbine + platinum; Vin + Pla + Endo: Vinorelbine + platinum + Endostar; Gem + Bev: Gemcitabine + bevacizumab; $\mathrm{Pla}+$ Pem + Endo: Platinum + pemetrexed + Endostar.

Acknowledgements

Not applicable. 


\section{Authors' contributions}

$\mathrm{PL}$ and $\mathrm{JZ}$ designed this study. $\mathrm{YL}, \mathrm{YY}$ and $\mathrm{AL}$ contributed equally to literature retrieval, literature screening, data extaction, quality assessment and statistical analysis. All authors resolved any disputes or inconsistencies through the discussion. All authors accepted the eventual manuscript. All authors read and approved the final manuscript.

\section{Funding}

Not applicable.

\section{Availability of data and materials}

The datasets analyzed during the current study are available from the corresponding author on reasonable request.

\section{Ethics approval and consent to participate}

Not applicable.

\section{Consent for publication}

Not applicable.

\section{Competing interests}

The authors declare that they have no competing interests.

Received: 8 August 2020 Accepted: 2 November 2020

Published online: 10 November 2020

\section{References}

1. Molina JR, Yang P, Cassivi SD, Schild SE, Adjei AA. Non-small cell lung cancer: epidemiology, risk factors, treatment, and survivorship. Mayo Clin Proc. 2008;83(5):584-94.

2. Folkman J. Tumor angiogenesis: therapeutic implications. N Engl J Med. 1971;285(21):1182-6.

3. Hanahan D, Weinberg RA. Hallmarks of cancer: the next generation. Cell. 2011;144(5):646-74.

4. Viallard C, Larrivée B. Tumor angiogenesis and vascular normalization: alternative therapeutic targets. Angiogenesis. 2017;20(4):409-26.

5. Carmeliet $P$, Jain RK. Molecular mechanisms and clinical applications of angiogenesis. Nature. 2011;473(7347):298-307.

6. Ferrara N, Hillan KJ, Gerber HP, Novotny W. Discovery and development of bevacizumab, an anti-VEGF antibody for treating cancer. Nat Rev Drug Discov. 2004;3(5):391-400

7. Alevizakos M, Kaltsas S, Syrigos KN. The VEGF pathway in lung cancer. Cancer Chemother Pharmacol. 2013;72(6):1169-81.

8. Sandler A, Gray R, Perry MC, et al. Paclitaxel-carboplatin alone or with bevacizumab for non-small-cell lung cancer. N Engl J Med. 2006;355(24):2542-50.

9. Cohen MH, Gootenberg J, Keegan P, Pazdur R. FDA drug approval summary: bevacizumab (Avastin) plus Carboplatin and Paclitaxel as first-line treatment of advanced/metastatic recurrent nonsquamous non-small cell lung cancer. Oncologist. 2007;12(6):713-8.

10. O'Reilly MS, Boehm T, Shing Y, et al. Endostatin: an endogenous inhibitor of angiogenesis and tumor growth. Cell. 1997;88(2):277-85.

11. Karamouzis MV, Moschos SJ. The use of endostatin in the treatment of solid tumors. Expert Opin Biol Ther. 2009;9(5):641-8.

12. Kim YM, Hwang S, Kim YM, et al. Endostatin blocks vascular endothelial growth factor-mediated signaling via direct interaction with KDR/FIk-1. J Biol Chem. 2002;277(31):27872-9.

13. Song HF, Liu XW, Zhang HN, Zhu BZ, Yuan SJ, Liu SY, et al. Pharmacokinetics of His-tag recombinant human endostatin in Rhesus monkeys. Acta Pharmacol Sin. 2005;26(1):124-8.

14. Wang J, Sun Y, Liu Y, Yu Q, et al. Results of randomized, multicenter, double-blind phase III trial of rh-endostatin $(\mathrm{YH}-16)$ in treatment of advanced non-small cell lung cancer patients. Zhongguo Fei Ai Za Zhi. 2005:8(4):283-90.

15. An J, Lv W. Endostar (rh-endostatin) versus placebo in combination with vinorelbine plus cisplatin chemotherapy regimen in treatment of advanced non-small cell lung cancer: a meta-analysis. Thorac Cancer. 2018:9(5):606-12.
16. Tierney JF, Stewart LA, Ghersi D, Burdett S, Sydes MR. Practical methods for incorporating summary time-to-event data into meta-analysis. Trials. 2007:8:16.

17. Higgins JP, Altman DG, Gøtzsche PC, et al. The Cochrane Collaboration's tool for assessing risk of bias in randomised trials. BMJ. 2011:343:d5928.

18. Wells G, Shea B, O'Connell D, et al. The Newcastle-Ottawa Scale (NOS) for assessing the quality of nonrandomised studies in meta-analyses. https://www.ohri.ca/programs/clinical_epidemiology/oxford.asp. Accessed 5 May 2016.

19. Gelman A, Rubin D. Inference from iterative simulation using multiple sequences. Statist Sci. 1992;7:457-511.

20. Zarogoulidis P, Kontakiotis T, Karaiskos T, et al. Docetaxel-carboplatin in combination with erlotinib and/or bevacizumab in patients with nonsmall cell lung cancer[J]. Oncotargets Therapy. 2013;2013:125-34.

21. Fukuda M, Kitazaki T, Ogawara D, et al. Randomized phase II study of pemetrexed or pemetrexed plus bevacizumab for elderly patients with previously untreated non-squamous non-small cell lung cancer: results of the Lung oncology group in Kyushu (LOGIK1201). Lung Cancer. 2019;132:1-8.

22. Galetta D, Cinieri S, Pisconti S, et al. Cisplatin/Pemetrexed followed by maintenance Pemetrexed Versus Carboplatin/Paclitaxel/Bevacizumab followed by maintenance bevacizumab in advanced nonsquamous lung cancer: the GOIM (Gruppo Oncologico Italia Meridionale) ERACLE Phase III Randomized Trial. Clin Lung Cancer. 2015;16(4):262-73.

23. Grønberg BH, Bremnes RM, Fløtten O, et al. Phase III study by the Norwegian lung cancer study group: pemetrexed plus carboplatin compared with gemcitabine plus carboplatin as first-line chemotherapy in advanced non-small-cell lung cancer. J Clin Oncol. 2009:27(19):3217-24

24. Han B, Xiu Q, Wang H, et al. A multicenter, randomized, double-blind, placebo-controlled study to evaluate the efficacy of paclitaxel-carboplatin alone or with endostar for advanced non-small cell lung cancer. J Thorac Oncol. 2011;6(6):1104-9.

25. Johnson DH, Fehrenbacher L, Novotny WF, et al. Randomized phase Il trial comparing bevacizumab plus carboplatin and paclitaxel with carboplatin and paclitaxel alone in previously untreated locally advanced or metastatic non-small-cell lung cancer. J Clin Oncol. 2004:22(11):2184-91.

26. Niho S, Kunitoh H, Nokihara H, et al. Randomized phase II study of firstline carboplatin-paclitaxel with or without bevacizumab in Japanese patients with advanced non-squamous non-small-cell lung cancer. Lung Cancer. 2012:76(3):362-7.

27. Patel JD, Socinski MA, Garon EB, et al. PointBreak: a randomized phase III study of pemetrexed plus carboplatin and bevacizumab followed by maintenance pemetrexed and bevacizumab versus paclitaxel plus carboplatin and bevacizumab followed by maintenance bevacizumab in patients with stage IIIB or IV nonsquamous non-small-cell lung cancer. J Clin Oncol. 2013;31(34):4349-57.

28. Rodrigues-Pereira J, Kim JH, Magallanes $M$, et al. A randomized phase 3 trial comparing pemetrexed/carboplatin and docetaxel/carboplatin as first-line treatment for advanced, nonsquamous non-small cell lung cancer. J Thorac Oncol. 2011;6(11):1907-14.

29. Reck M, von Pawel J, Zatloukal P, et al. Phase III trial of cisplatin plus gemcitabine with either placebo or bevacizumab as first-line therapy for nonsquamous non-small-cell lung cancer: AVAil. J Clin Oncol. 2009;27(8):1227-34

30. Scagliotti GV, Parikh P, von Pawel J, et al. Phase III study comparing cisplatin plus gemcitabine with cisplatin plus pemetrexed in chemotherapynaive patients with advanced-stage non-small-cell lung cancer. J Clin Oncol. 2008;26(21):3543-51.

31. Scagliotti GV, De Marinis F, Rinaldi M, et al. Phase III randomized trial comparing three platinum-based doublets in advanced non-small-cell lung cancer. J Clin Oncol. 2002;20(21):4285-91.

32. Schiller JH, Harrington D, Belani CP, et al. Comparison of four chemotherapy regimens for advanced non-small-cell lung cancer. N Engl J Med. 2002;346(2):92-8

33. Smit EF, van Meerbeeck JP, Lianes $P$, et al. Three-arm randomized study of two cisplatin-based regimens and paclitaxel plus gemcitabine in advanced non-small-cell lung cancer: a phase III trial of the European Organization for Research and Treatment of Cancer Lung Cancer GroupEORTC 08975. J Clin Oncol. 2003:21(21):3909-17. 
34. Soria JC, Márk Z, Zatloukal P, et al. Randomized phase II study of dulanermin in combination with paclitaxel, carboplatin, and bevacizumab in advanced non-small-cell lung cancer. J Clin Oncol. 2011;29(33):4442-51.

35. Spigel DR, Anthony Greco F, Waterhouse DM, et al. Phase II trial of ixabepilone and carboplatin with or without bevacizumab in patients with previously untreated advanced non-small-cell lung cancer. Lung Cancer. 2012;78(1):70-5.

36. Spigel DR, Hainsworth JD, Joseph MJ, et al. Randomized phase 2 trial of pemetrexed, pemetrexed/bevacizumab, and pemetrexed/carboplatin/ bevacizumab in patients with stage IIIB/IV non-small cell lung cancer and an Eastern Cooperative Oncology Group performance status of 2. Cancer. 2018;124(9):1982-91.

37. Treat JA, Gonin R, Socinski MA, et al. A randomized, phase III multicenter trial of gemcitabine in combination with carboplatin or paclitaxel versus paclitaxel plus carboplatin in patients with advanced or metastatic nonsmall-cell lung cancer. Ann Oncol. 2010;21(3):540-7.

38. Wu YL, Lu S, Cheng Y, et al. Efficacy and safety of pemetrexed/cisplatin versus gemcitabine/cisplatin as first-line treatment in Chinese patients with advanced nonsquamous non-small cell lung cancer. Lung Cancer. 2014;85(3):401-7.

39. Zinner RG, Obasaju CK, Spigel DR, et al. PRONOUNCE: randomized, open-label, phase III study of first-line pemetrexed + carboplatin followed by maintenance pemetrexed versus paclitaxel + carboplatin + bevacizumab followed by maintenance bevacizumab in patients with advanced nonsquamous non-small-cell lung cancer. J Thorac Oncol. 2015;10(1):134-42.

40. Zhao X, Mei K, Cai X, et al. A randomized phase II study of recombinant human endostatin plus gemcitabine/cisplatin compared with gemcitabine/cisplatin alone as first-line therapy in advanced non-small-cell lung cancer. Invest New Drugs. 2012;30(3):1144-9.

41. Zhou C, Wu YL, Chen G, et al. BEYOND: a randomized, double-blind, placebo-controlled, Multicenter, phase III study of first-line carboplatin/ paclitaxel plus bevacizumab or placebo in chinese patients with advanced or recurrent nonsquamous non-small-cell lung cancer. J Clin Oncol. 2015;33(19):2197-204.

42. Marinis DE, F, Bidoli P, Luciani A, et al. EAGLES study: first-line bevacizumab in combination with chemotherapy in elderly patients with advanced, metastatic, non-squamous non-small cell lung cancer. Anticancer Res. 2017:37(5):2457-64.

43. Yu X, Zhang $L$, Chen J. Effectiveness of treatment with endostatin in combination with emcitabine, carboplatin, and gemcitabine in patients with advanced non-small cell lung cancer: a retrospective study. Open Med. 2018;13:142-7.

44. Schuette W, Schneider CP, Engel-Riedel W, et al. 65Plus: open-label study of bevacizumab in combination with pemetrexed or pemetrexed/carboplatin as first-line treatment of patients with advanced or recurrent nonsquamous non-small-cell lung cancer. Lung Cancer. 2017;8:217-29.

45. Zhou S, Zuo L, He X, Pi J, Jin J, Shi Y. Efficacy and safety of rh-endostatin (Endostar) combined with pemetrexed/cisplatin followed by rhendostatin plus pemetrexed maintenance in non-small cell lung cancer: a retrospective comparison with standard chemotherapy. Thorac Cancer. 2018;9(11):1354-60.

46. Liu ZJ, Wang J, Wei XY, et al. Predictive value of circulating endothelial cells for efficacy of chemotherapy with Rh-endostatin in non-small cell lung cancer. J Cancer Res Clin Oncol. 2012;138(6):927-37.

47. Sun Y, Wang JW, Liu YY, et al. Long-term results of a randomized, doubleblind, and placebo-controlled phase III trial: Endostar (rh-endostatin) versus placebo in combination with vinorelbine and cisplatin in advanced non-small cell lung cancer. Thorac Cancer. 2013:4(4):440-8.

\section{Publisher's Note}

Springer Nature remains neutral with regard to jurisdictional claims in published maps and institutional affiliations.
Ready to submit your research? Choose BMC and benefit from:

- fast, convenient online submission

- thorough peer review by experienced researchers in your field

- rapid publication on acceptance

- support for research data, including large and complex data types

- gold Open Access which fosters wider collaboration and increased citations

- maximum visibility for your research: over $100 \mathrm{M}$ website views per year

At BMC, research is always in progress.

Learn more biomedcentral.com/submissions 